2018-10

\title{
A review of uncertainty analysis in building energy assessment
}

\section{Tian, W}

http://hdl.handle.net/10026.1/11542

10.1016/j.rser.2018.05.029

Renewable and Sustainable Energy Reviews

Elsevier

All content in PEARL is protected by copyright law. Author manuscripts are made available in accordance with publisher policies. Please cite only the published version using the details provided on the item record or document. In the absence of an open licence (e.g. Creative Commons), permissions for further reuse of content should be sought from the publisher or author. 


\section{A review of uncertainty analysis in building energy assessment}

Wei Tian ${ }^{\mathrm{a}, \mathrm{b} *}$, Yeonsook Heo ${ }^{\mathrm{c}}$, Pieter de Wilde ${ }^{\mathrm{d}}$, Zhanyong $\mathrm{Li}^{\mathrm{a}, \mathrm{b}}$, Da Yan ${ }^{\mathrm{e}}$, Cheol Soo Park ${ }^{\mathrm{f}}$, Xiaohang Feng ${ }^{\mathrm{e}}$, and Godfried Augenbroe ${ }^{\mathrm{g}}$

${ }^{a}$ Tianjin Key Laboratory of Integrated Design and On-line Monitoring for Light Industry \& Food Machinery and Equipment, College of Mechanical Engineering, Tianjin University of Science and Technology, Tianjin 300222, China

${ }^{\mathrm{b}}$ Tianjin International Joint Research and Development Center of Low-Carbon Green Process Equipment, Tianjin 300222, China

${ }^{\mathrm{c}}$ Department of Architecture, University of Cambridge, Cambridge, UK

${ }^{\mathrm{d}}$ Chair of Building Performance Analysis, Environmental Building Group, University of Plymouth, Plymouth, Devon PL4 8AA, UK

${ }^{\mathrm{e}}$ School of Architecture, Tsinghua University, Beijing 100084, China

${ }^{\mathrm{f}}$ Department of Architecture and Architectural Engineering, College of Engineering, Seoul National University, Seoul, 08826, South Korea

${ }^{\mathrm{g}}$ College of Architecture, Georgia Institute of Technology, Atlanta, USA

*Corresponding author: Wei Tian, Tel: +86 (022) 60600705 , Email: tjtianjin@ gmail.com

Abstract: Uncertainty analysis in building energy assessment has become an active research field because a number of factors influencing energy use in buildings are inherently uncertain. This paper provides a systematic review on the latest research progress of uncertainty analysis in building energy assessment from four perspectives: uncertainty data sources, forward and inverse methods, application of uncertainty analysis, and available software. First, this paper describes the data sources of uncertainty in building performance analysis to provide a firm foundation for specifying variations of uncertainty factors affecting building energy. The next two sections focus on the forward and inverse methods. Forward uncertainty analysis propagates input uncertainty through building energy models to obtain variations of energy use, whereas inverse uncertainty analysis infers unknown input factors through building energy models based on energy data and prior information. For forward analysis, three types of approaches (Monte Carlo, non-sampling, and non-probabilistic) are discussed to provide sufficient choices of uncertainty methods depending on the purpose and specific application of a building project. For inverse analysis, recent research has concentrated more on Bayesian computation because Bayesian inverse methods can make full use of prior information on unknown variables. Fourth, several applications of uncertainty analysis in building energy assessment are discussed, including building stock analysis, HVAC system sizing, variations of sensitivity indicators, and optimization under uncertainty. Moreover, the software for uncertainty analysis is described to provide flexible computational environments for implementing uncertainty methods described in this review. This paper concludes with the trends and recommendations for further research to provide more convenient and robust uncertainty analysis of building energy. Uncertainty analysis has been ready to become the mainstream approach in building energy assessment although a number of issues still need to be addressed.

Keywords: building energy; uncertainty analysis; uncertainty propagation; inverse problems; Bayesian computation 


\section{Highlights}

- A comprehensive review on uncertainty analysis in building energy assessment is presented.

- A reliable dataset for uncertain factors in buildings needs to be constructed.

- Further research on quantifying stochastic occupant behaviours in buildings is needed.

- The Monte Carlo method is the most widely used uncertainty method in building energy analysis.

- Bayesian computation receives more attention in calibrating building energy models. 

Abbreviations
ACOSSO
Adaptive COmponent Selection and Smoothing Operator
ARIMA
Auto-Regressive Integrated Moving Average
ASHRAE
American Society of Heating, Refrigerating and Air-Conditioning Engineers
BUGS
Bayesian inference Using Gibbs Sampling
$\mathrm{CDF}$
Cumulative Distribution Function
DST
Demster-Shafer Theory
GIS
Geographical Information System
GP
Gaussian Process
GURA-W
Georgia Tech Uncertainty and Risk Analysis Workbench
HVAC
Heating, Ventilation, and Air Conditioning
IDF
Input Data File
LES
Least Square Estimation
LHS
Latin Hyper-cube Sampling
MARS
Multivariate Adaptive Regression Splines
MCMC
Markov Chain Monte Carlo
MLE
Maximum likelihood estimation
NREL
National Renewable Energy Laboratory
RBDO
Reliability Based Design Optimization
RDO
Robust Design Optimization
SMS-EMOA
S Metric Selection - Evolutionary Multi-objective Optimisation Algorithm
SRC
Standardized Regression Coefficient
SRRC
Standardized Rank Regression Coefficients
TMY
Typical Meteorological Year
UKCP09
UK Climate Projections
UQ
Uncertainty Quantification 
Uncertainty analysis has received increasing attention in the field of building energy analysis [1-4] because a number of variables that influence building thermal performance are inherently uncertain, such as occupant behaviour, thermal properties of building envelope, and weather conditions [5, 6]. Moreover, the development of modern uncertainty quantification techniques provides more advanced methods and tools to facilitate the research on uncertainty analysis for a better understanding of the nature of building energy and associated energy models $[7,8]$. Therefore, uncertainty analysis has been widely implemented in various areas of building energy analysis, including model calibration [1,9], life cycle analysis [10-12], building stock analysis $[13,14]$, impact $\&$ adaptation to climate change $[15,16]$, sensitivity analysis $[17$, $18]$, spatial analysis [19, 20], and optimization [21, 22].

Uncertainty analysis in building energy assessment can be divided into two categories as shown in Figure 1: forward and inverse uncertainty quantification [23-25]. Forward uncertainty analysis (also called uncertainty propagation) focuses on quantifying the uncertainty in the system outputs propagated from uncertain input variables through mathematical models, while the purpose of inverse uncertainty analysis (also called model calibration) determines unknown variables through mathematical models from measurement data. From the perspective of building energy analysis, forward uncertainty quantification can predict energy use or carbon emissions using building energy models with input variations, whereas inverse uncertainty quantification can quantify unknown input variations through building energy models after collecting energy data from buildings. To date, considerably more research has been carried out on forward uncertainty propagation than on inverse uncertainty quantification in the field of building energy analysis. This is not surprising, as inverse uncertainty quantification is significantly more difficult than forward uncertainty propagation. Nevertheless, forward and inverse uncertainty analyses are closely linked [8]. Efficient forward uncertainty propagation is necessary for inverse uncertainty analysis because sampling-based inverse uncertainty analysis usually involves a large number of simulation runs [1]. The results from inverse uncertainty analysis are often used for forward uncertainty propagation to predict building energy use from various energy saving strategies $[9,26]$.

A distinction is often made between two types of uncertainty: aleatory uncertainty and epistemic uncertainty [27, 28]. Aleatory uncertainty (also called variability, stochastic, irreducible, and type A uncertainty) is due to inherent or natural variation of the system under investigation. In contrast, epistemic uncertainty (also called state of knowledge, subjective, reducible, and type B uncertainty) arises from a lack of knowledge. In building energy analysis, an example of aleatory uncertainty is occupancy presence, which can be better characterized from additional experiments or observation, but not be reduced as it is fundamentally impossible to predict variations of occupancy patterns for the future. Examples of epistemic uncertainty include lighting and appliance power densities, which can be better quantified by collecting more information to reduce its uncertainty, such as by installing measurement equipment for lighting and appliances. Note that not all of these uncertainties can be represented as specific probability functions (such as normal distribution, Gamma distribution, and uniform distribution). Aleatory uncertainty is naturally treated in a probabilistic framework, whereas epistemic uncertainty may be specified in a probabilistic or non-probabilistic way, including second order probability, interval, evidence theory, and fuzzy sets [28].

Researchers in the field of building energy simulation have proposed several classifications of uncertainty to represent the different characteristics of uncertainty in building energy analysis $[3,22,29,30]$. Uncertainty can be divided into model form uncertainty and parameter uncertainty [31, 32]. Model form uncertainty (also called model discrepancy) refers to underlying the missing physics, numerical approximation, and other issues of computer 
programs [7, 29], whereas parameter uncertainty refers to uncertainty associated with the values of parameter that appear in building energy simulation models. Uncertain parameters in building energy analysis can be further divided into three categories: design parameters, inherent uncertain parameters, and scenario parameters [3, 22, 30, 33-35]. Uncertainty in design parameters exists in the design process where design parameters are determined through a series of design stages. For example, while the exact insulation materials or window types are not known in the early design stage, they will become known during the detailed design stage. Inherent uncertain parameters are usually uncontrollable, such as occupant behaviour, or the deviations between rated and actual plant system efficiencies. Scenario parameters refer to potentially varying economic or climatic conditions. Inherent uncertain parameters are usually denoted by normal distributions, whereas design uncertainty can be expressed by continuous or discrete uniform distributions [36]. Ramallo-González et al. [22] subdivided inherent uncertain parameters into workmanship \& quality of building elements and occupant behaviour. Note that fewer studies have been carried out on model form uncertainty than on parameter uncertainty in the area of building energy analysis [37].

Although a large number of studies have been conducted on uncertainty analysis of building energy analysis, a comprehensive up-to-date review on uncertainty analysis in the area of building energy assessment is still unavailable. Therefore, this paper aims to provide a detailed systematic overview of uncertainty analysis in building energy assessment from four aspects: uncertainty data sources, both forward and inverse uncertainty methods, application of uncertainty analysis, and available software for uncertainty analysis. Because reliable uncertainty data are the foundation for uncertainty analysis in building energy analysis, it is necessary to gain a good understanding of the latest research development of data sources relevant to building performance as will be described in section 3. Forward and inverse analysis (as will be described in section 4 and section 5, respectively) are the two main methods applied in the field of building performance assessment. The forward uncertainty analysis will discuss the Monte Carlo sampling-based, non-sampling, and non-probabilistic approaches to help an analyst choose the appropriate method. Inverse uncertainty analysis will be described from the perspectives of both the frequentist and Bayesian methods to provide a full picture of modern statistics to deal with the calibration problems of building energy models. Four applications on uncertainty analysis in building energy performance will be described in section 6 , including building stock analysis, HVAC system sizing, variations of indicators of sensitivity analysis, and optimization under uncertainty. The software available for uncertainty analysis will be presented in section 7 to provide the computation environment for implementing the uncertainty methods described in this paper. Moreover, the trends and recommendations for further research will be summarized in the final section to provide more convenient and robust uncertainty analysis in assessing building energy performance. It should be emphasized that uncertainty analysis is not yet regarded as standard practise in assessing building performance in industry although the traditional deterministic approach is considered to be unacceptable due to the lack of sufficient information obtained from one single simulation run $[38,39]$. This review will also help to promote uncertainty analysis as a mainstream method in the area of building performance assessment.

\section{Methodology}

This literature review follows the concept-centric principle proposed by Webster and Watson [40] and also incorporates the strengths of reviews published in the field of building energy analysis [41-43]. The research methodology is composed of three steps as follows.

(1) Search and Screen relevant research papers. A keyword search was conducted using Google Scholar and Scopus database since these databases can cover broad publications in the 
field of building energy analysis. All the journal or conference papers should meet the following criteria: papers written in English; peer-reviewed publications; relevant to the topics as described in the last paragraph of "Introduction" section; more focused on recent research findings. The online publications from the IBPSA (International Building Performance Simulation Association) with a larger number of international and regional conference papers are also used to select papers relevant to this overview since this online database includes the latest and important findings in the field of building energy analysis.

(2) Review all relevant publications. All the papers selected in the first step will be reviewed carefully by the authors from four aspects: uncertainty data sources, forward $\&$ inverse uncertainty approaches, application of uncertainty analysis, and available software in this field.

(3) Identify gaps and future research directions. After carefully analysing relevant papers, research trends and gaps are identified in uncertainty analysis of building energy performance. Moreover, future research directions are also determined to make uncertainty analysis become a main-stream method in building energy assessment.

\section{Sources of uncertainty in building performance analysis}

In this section, four types of uncertainty are summarized, as listed in Table 1: weather data, building envelope, HVAC system, and occupant behaviour. In the area of uncertainty analysis of building energy, two initial studies on quantifying uncertainty parameters are performed by Macdonald [44] and de Wit [45]. Macdonald [44] quantified three types of input parameters based on an extensive literature review: thermo-physical properties, casual gains, and infiltration rates. In addition to these parameters, de Wit [45] quantified uncertain parameters with little measurement data available, including wind pressure coefficients and indoor air temperature distributions. Recently, Sun [32] provided more information on uncertainty of ground albedo, convective heat transfer coefficients, and lighting/plug loads. In Wang's thesis [31], more attention is concentrated on infiltration, workmanship issues related to thermal bridges, occupancy variables, and HVAC system uncertainty. These recent works are included in a full repository of quantified sources of uncertainty as part of the Georgia Tech Uncertainty and Risk Analysis-Workbench (GURA-W) developed by the Georgia Institute of Technology [37], which will be described in section 4.1.3. De Wit [45], Heo [46], and Struck [47] provide more general descriptions of uncertain parameters.

\subsection{Weather data}

Due to changeable nature of weather, weather data is highly uncertain, causing variation of energy use in buildings. In building energy simulation, a typical meteorological year chosen from actual weather data over several decades is usually applied using the Finkelstein-Schafer method $[16,48]$. Currently, increasing concern is being shown that a single weather data file (typically containing 8760 hourly values) cannot contain reliable and sufficient information on plausible weather conditions and their likelihoods in assessing building energy performance [49, 50]. Simulated energy consumption using typical weather data does not necessarily represent the average energy use based on the weather data of actual historical meteorological years. Moreover, building performance is affected by future climate, not by historical weather conditions. Most weather data derived from historical data do not represent the likely long-term weather conditions to which buildings will be exposed in the future. These two aspects (historical and future weather data) are discussed in the following subsections. 


\subsubsection{Historical weather data}

Two methods are used to determine variations of historical weather data in building energy analysis (as an alternative to using only one typical weather data file). In the first method, several weather files are used, such as the typical year file, cold weather data, and hot weather data, to sufficiently include the possible variations in the weather conditions with the minimal weather dataset. Rodríguez et al. [51] used three weather files to represent the uncertainty of weather data for the extreme cold, medium, and extreme hot years. Breesch and Janssens [52] applied two weather files to consider the effects of warming summer conditions, including normal and warm temperatures.

In the second method, all the recent weather data are used to represent long-term weather uncertainty according to the frequency of occurrence [53, 54]. Hong et al. [49] assessed the weather impact on building energy performance using 30-year (1980-2009) historical weather data. They implemented a total of 3162 simulation runs by considering three types of office buildings with two design efficiency levels in all 17 ASHRAE climate zones. They found that the typical meteorological year (TMY3) weather file was not able to represent the average energy performance predicted by an actual 30-year weather dataset. Wang et al. [55] used the weather files of 10-15 years to assess the variations of energy use in an office building, and found that the energy variations ranged from $-4.0 \%$ to $6.1 \%$ according to the weather file, compared with the energy prediction using the TMY data. Four USA cities were used as locations to represent variations of climate types: Washington DC, Chicago, Atlanta, and San Francisco. Sun et al. [56] used 32 weather datasets (1982-2013) to investigate the effect of weather uncertainties on sizing HVAC system under different climate zones. As an alternative to directly using the historical weather data, Lee et al. [57] developed a stochastic model based on a Vector Auto-Regressive process in which a number of varying weather conditions are generated based on a historical weather dataset.

Most weather data are measured at a nearby meteorological station, which cannot represent actual microclimate conditions around a building. Hence, meteorological weather data need to be modified to reflect the impact of nearby vegetation, neighbouring buildings, and mesoscale flow characteristics. Sun et al. [58] built statistical models to quantify the uncertainty of four microclimate variables: local wind speed, local temperature, wind pressure and solar irradiation.

Uncertainty in the weather data has been handled in different ways depending on the types of design applications and modelling methods. For designing mechanical systems, Huang et al. [35] implemented normal distribution to quantify uncertainty in both ambient temperature and relative humidity values selected as the design weather conditions for calculating peak cooling loads. By using a quasi-steady-state calculation method for building energy rating, Corrado and Mechri [59] used a bivariate normal distribution to quantify uncertainty in both monthly average temperatures and global horizontal solar radiations, since these two weather variables are highly correlated.

\subsubsection{Future weather data}

A large number of studies have been conducted on assessing the impact and adaptation to climate change in buildings $[15,16,60-62]$. Climate projections from global circulation models usually have larger spatial and temporal resolutions (typical $300 \mathrm{~km} \mathrm{X} 300 \mathrm{~km}$ and $24 \mathrm{~h}$ ) than the local hourly data required for dynamic building energy simulation [63]. Therefore, these weather projections need to be downscaled using suitable methods, including dynamical downscaling, stochastic weather generation, interpolation, and morphing approach. The morphing approach widely used in building energy analysis is to shift and stretch weather 
variables from the current weather time series in order to create new future weather data by encapsulating information about the future climate $[16,64]$. The advantages of this method are that the future weather sequences are meteorologically consistent with the best climate projection.

A major step towards quantifying the uncertainty of future climate is the UK Climate Projections (UKCP09) released in 2009 [65]. UKCP09 deals with three types of uncertainty: complexity of climate system, natural climate variability, variations of future pathway of greenhouse gas and aerosol emissions. For the uncertainty of greenhouse gas emissions, the UKCP09 applies three scenarios: low, medium, and high [65]. For each scenario, the UKCP09 provides weather projections in a probabilistic manner by accounting for the complexity and natural variability of the climate system for a range of possible outcomes, which makes riskbased analysis easier in decision making process. Since a large number of hourly weather files can be generated from UKCP09, further developments have concentrated on finding ways to efficiently implement the UKCP09 in building energy analysis [61, 62].

\subsection{Building envelope}

The parameters related to building envelope have been categorized into three types: thermal properties, surface properties, and other parameters. Compared to occupant behaviour and HVAC systems in buildings, there has been more data available on the uncertainty of building envelope.

\subsubsection{Thermal properties}

An early extensive document on the thermal properties of building materials was compiled by Clarke et al. [66]. Their report reviewed the available data and described the variations of thermal property values in these data. Based on these data, Macdonald [44] derived more detailed uncertainty data of thermal properties, which can be easily applied to uncertainty analysis. Detailed information on the uncertainty ranges of the thermal properties of building materials was presented in Macdonald's studies [44], including conductivity, density, and specific heat capacity. These data have been widely used in the uncertainty analysis of building energy use $[35,52,59]$. Note that the uncertainty ranges of thermal properties are due to both measurement errors and environmental conditions, such as moisture, temperature, and age. For example, moisture conditions have simultaneous effects on density, thermal conductivity, and heat capacity. Dominguez-Munoz et al. [67] provided more specific information on the thermal conductivity of insulation materials using several hundred measurements of conductivity from seven European national laboratories.

\subsubsection{Surface properties}

The surface properties of building envelope affect building energy performance by absorbing or reflecting solar energy and emitting thermal energy. Macdonald [44] summarized the mean and standard deviation of emissivity and absorptivity values for different building materials based on the work by Clarke et al. [66]. The emissivity of materials often used in building construction is around 0.9-0.95 [68] and the corresponding standard deviation is 0.02 [44]. A typical emissivity value for highly polished materials (such as aluminium foil) is 0.05 [68] and its standard deviation is 0.01 [44]. For the solar absorptivity of bricks, the average values are 0.49 and 0.76 for light and dark bricks, respectively, and their standard deviation is 0.04 [44].

Ground reflectance (also called ground reflectivity or albedo) is an often overlooked 
parameter in building energy analysis [69], and can differ depending on surface properties, cloud cover, snow conditions, and other factors. Based on Thevenard et al. [69], Silva and Ghisi [33] used a triangular distribution with a range from 0.13 to 0.26 for the reflectance of ground surface. Sun et al. [58] obtained a non-normal distribution of ground reflectance derived from various available data sources using the Monte Carlo method. In their research, the distribution of ground reflectance from their research is clustered at about 0.25 and ranges from 0.05 to 0.45 in the case of no snow for city terrain. In the presence of snow, the ground reflectance increases significantly, ranging from approximately 0.75 to 0.95 for fresh snow cover $[58,69]$.

\subsubsection{Other parameters}

This section focuses on infiltration rate, thermal bridges, convective heat transfer coefficient, and thickness of building materials.

Infiltration rate (closely related to air tightness) is a function of age, construction quality, building use, and weather conditions [9, 44]. Infiltration rate is one of the most uncertain parameters since it is difficult to measure in buildings. Moreover, in previous studies, the infiltration rate has been ranked as one of the key variables influencing building energy use based on sensitivity analyses $[9,70]$. Most papers have quantified uncertainty in the infiltration rate on the basis of a collection of measurement data available from existing buildings $[9,66$, 71, 72]. Emmerich et al. [73] summarised an extensive dataset of existing fan pressurized tests, most of which were sourced from the work by Persily [74]. Li et al. [75] studied the natural ventilation and infiltration rate in the dormitory and analysed the uncertainty.

Unwanted heat transfer occurs through thermal bridges in a building at connections between envelope components such as wall/window and wall/floor connections. The severity of this thermal bridge effects depends on construction details and the quality of construction workmanship [76]. Moon [76] quantified the effect of construction details on the temperature factor based on simulation results of three case studies. He also quantified the effect of poor workmanship on the temperature factor by comparing simulation results with measurements. This approach was adopted in the Moon's paper [71] to investigate the effect of varying quality of thermal bridge workmanship and infiltration rates on the building energy demand.

Internal and external convective heat transfer coefficients have been studied extensively in the field of building energy assessment $[26,32]$. In these studies, external convective heat transfer coefficients are often expressed as a function of local wind speed and surface properties (e.g., roughness). However, a few studies have been carried out on quantifying the uncertainty of convective heat transfer coefficients in buildings. Sun [32] summaries correlation relationships between two empirical coefficients derived in the previous studies for the calculation of external heat transfer coefficients, using a bivariate normal distribution, since these two coefficients are found to be correlated. Huang et al. [35] used a normal distribution and a triangular distribution for an internal and external convection heat transfer rate, respectively.

Uncertainty in the thickness of building materials is caused by the differences between design specifications and construction outcomes. Silva and Ghisi [33] assumed that the material thickness parameter follows a normal distribution with a standard deviation of 10 percent of its mean value. The same uncertainty range is also used for material thickness parameters in the research by Hopfe and Hensen [3].

\subsection{HVAC system}

Compared to other types of uncertainty in buildings, few studies have been conducted on the uncertainty related to HVAC systems [55, 77]. Building energy analysis usually assumes 
that HVAC systems operate in ideal conditions. In reality, however, the performance of HVAC systems is affected by a number of factors, such as oversizing, ageing, maintenance, usual wear and tear.

Typically simulation studies have considered only uncertainty in mechanical system efficiencies, quantified as the form of a probability distribution $[9,51]$. Beyond the system efficiencies, Wang et al. [55] explored the uncertainty of energy use as the result of different levels of building operation strategies that include lighting control, plug-in equipment control, HVAC operation schedule, variable-air-volume box minimum-flow, economizer setting, night setback, supply air temperature control, and temperature setting for non-occupied hours. The results suggest the uncertainty in the annual energy use ranges from $-28.7 \%$ to $79.2 \%$ due to the parametric variations associated with building operation strategies. Yan et al [78] quantified uncertainty associated with actual system operation, specifically the outdoor airflow control parameter, with the application of HVACSIM+ simulations and measured flow rates.

Another relevant topic is the longitudinal performance of HVAC systems as the result of system deterioration over time. The National Renewable Energy Laboratory (NREL) report [79] provides typical degradation rates depending on the maintenance level and system type; the degradation rate for boilers and constant/variable volume fans is $0.2 \%$ per year with good maintenance and $0.5 \%$ per year without maintenance. For central chillers and heat pumps, the degradation rate is $0.1 \%$ with maintenance and $1 \%$ per year without maintenance. In order to account for uncertainty associated with the degradation rate, de Wilde et al. [80] proposed a stochastic process model with a gamma distribution in order to model system deterioration over time. Huang et al. [81] proposed a method using a Bayesian Markov Chain Monte Carlo (MCMC) method to estimate the degradation effect of chiller systems. They demonstrated through a case study that the proposed method provides accurate predictions with associated uncertainty levels to evaluate the reliability of chillers in future years.

\subsection{Occupant behaviour}

The relationship between the occupant behaviour and thermal performance of buildings has recently become a very active research area [82-87]. Occupant behaviour is regarded as a major uncertainty source that can account for up to $30 \%$ of variation in building energy performance [88]. Studies have shown that occupant behaviour leads to the uncertainty in energy consumption even within the same building $[89,90]$. Previous researchers have studied various aspects of occupant behaviour in building, including occupant monitoring, ontology of occupant behaviour, behaviour model development \& evaluation, and model implementation [83, 91, 92].

Most current building energy simulation programs treat the variables associated with occupant behaviour as deterministic by allowing users to specify fixed temporal schedules for occupancy-related variables, such as occupants, lighting use, plug loads, and cooling/heating set-points. Such schedules are easy to implement, but do not represent the complex stochastic nature of human behaviour or its interaction with the indoor environment in buildings. New approaches have been developed to adequately present the variations of occupant behaviour, and these methods can be categorized into two types: implicit and explicit $[82,93]$. The implicit models focus on predicting occupant control actions associated with building systems (e.g. windows, lights, equipment) rather than understanding underlying logics behind occupant behaviour. In contrast, the explicit models directly represent behavioural logics to determine the state of the occupant and his/her control actions accordingly.

Implicit occupant models are widely used in the field of building energy analysis because they are a natural extension of the schedule-based approach currently used in building simulation programs $[44,70]$. Moreover, occupant presence may not be the best proxy for predicting internal loads (e.g., lighting and plug-in equipment) [94]. Corrado and Mechri [59] 
used a triangular distribution for the total number of occupants and a normal distribution for occupant metabolic rates. Ward et al. [94] compared the different models of internal loads, including Menezes [95], DEmand LOad REconStructor (DELORES) [96], Sun [32], and AutoRegressive Integrated Moving Average (ARIMA). Breesch and Janssens [52] implemented three scenarios (low, medium, and high) to represent the variations of internal heat gains.

Explicit models for occupants can directly determine the state of occupant behaviour in a building. This type of models include Markov chain [97], agent approach [98], Bernoulli process [99], and random walk [100, 101]. The Markov chain method is based on conditional probabilities derived from measurement data that yield stochastic predictions [97]. In contrast, the agent approach focuses on modelling the underlying complicated rules such as the interactions of occupants' perception, intention, etc. Bernoulli processes may be the most simplistic of stochastic models as it treats the probability of an event as being independent on the previous state. A random walk is a sequence of random variables obtained by adding a white noise to a time series data to simulate occupant behaviour in an unpredictable way [100]. According to [101], the occupancy pattern can significantly vary depending on the building type (process driven buildings such as offices, factories, residences vs. random walk buildings such as university labs, libraries), building environmental control (central autonomous HVAC vs. individual control), and many other unknowns. More research is needed in this area to better understand occupant behaviour and find appropriate ways to simulate these behaviour and associated uncertainty in building energy analysis.

\section{Forward uncertainty quantification in building performance analysis}

Uncertainty propagation can be divided into probabilistic and non-probabilistic methods [102]. Probabilistic uncertainty approaches are based on rigorous probability theory under the availability of sufficient data, whereas non-probabilistic approaches are developed to cope with a lack of information or data. Probabilistic uncertainty propagation can be further categorized into sampling-based and non-sampling approaches [103]. Sampling-based methods belong to external methods (also called non-intrusive), which treats an original deterministic model as a black-box model by running this deterministic model with different samples many times. In contrast, non-sampling methods include perturbation methods, moment equations, spectral representations (stochastic Galerkin and collocation) methods, and classical stochastic differential equations [103-105]. The advantage of internal (non-sampling) methods is their ability to provide high accuracy and high efficiency results, while this method requires extensively modifying the existing computational system code for uncertainty propagation [106]. The advantage of external uncertainty propagation is the ability to maintain a wellvalidated model code at a high computation cost.

Section 4.1 and 4.2 discuss the Monte Carlo simulation method and its variations, respectively. Section 4.3 and 4.4 presents non-sampling and non-probabilistic uncertainty propagation methods, respectively. The relevant references for both forward and inverse uncertainty analysis in building energy analysis are summarised in Table 2.

\subsection{Monte Carlo sampling-based simulation}

Monte Carlo-based simulation is the most widely used uncertainty propagation method in the area of building energy assessment [107-109]. This is because this method is very intuitive and easy to implement compared to other uncertainty propagation approaches (such as full factorial numerical integration, stochastic Galerkin, and discrete projection). Moreover, the samplingbased method is usually regarded as the most reliable uncertainty technique since it can be applied to most simulation environments and deal with different types of probability functions 
of input variables, even for correlated variables $[104,110]$. The main disadvantage of this method is the slow convergence rate with a large number of function evaluations, which incurs a high computational cost. The high computational cost because of using the Monte Carlo uncertainty simulation can be reduced in three ways. First, more efficient sampling methods can be used, such as Latin hypercube sampling or Sobol sequence, which is discussed in section 4.1.2. Second, surrogate models can be applied instead of original expensive engineering mathematical models in propagating uncertainty as is described in section 4.2.3. Third, more recent uncertainty quantification methods can be employed, such as stochastic polynomial chaos expansion, stochastic collocation. These new numerical methods are briefly described in section 4.3. For detailed description of these new methods, please refer to $[8,110]$.

The procedure for quantifying uncertainty in building performance using the typical Monte Carlo simulation method is illustrated in Figure 2. The first step involves specifying probability distributions of uncertain input variables. The second step is to generate samples for input variables using sampling methods. The third step involves running mathematical system models to obtain matrixes corresponding to input samples for output variables. The final step is to present uncertainty results of output variables. A further optional step can be used in which sensitivity analysis is conducted to determine the key factors for explaining the distributions of building thermal performance. These steps are described in detail in the following subsections.

\subsubsection{Specify distributions of input variables}

The first step in quantifying uncertainty in building performance using the typical Monte Carlo simulation method involves specifying the probabilistic distributions for all uncertain variables. This step is both the most important and the most difficult aspect of the uncertainty analysis of building energy performance. Section 3 provides a summary of the quantification of uncertainty distributions for input parameters. In the field of building energy analysis, the Gaussian distribution is the most widely used distribution for inherent parametric uncertainty [2, $6,35,36,45]$. Gaussian distributions need to be truncated where necessary to avoid unfeasible values (for instance, a negative value or zero for thermal resistance). In contrast, uniform distribution is commonly used in presenting the possible change of various building design strategies [111-114].

In the case of a large number of input variables, performing sensitivity analysis prior to implementing uncertainty analysis can significantly reduce computational cost by selecting key factors influencing building energy performance. Spitz et al [85] selected the ten most influential parameters from 139 parameters with respect to their effect on the air temperature. Kim [115] selected the first five important factors using the Standardized Rank Regression Coefficients (SRRC) method to construct regression models for forward uncertainty analysis. Dominguez-Munoz et al. [116] used the standardized regression coefficient (SRC) to determine the key factors affecting peak cooling loads. Tian and Choudhary [12] implemented SRC and multivariate adaptive regression splines (MARS) to identify four important variables for the energy performance of schools located in London.

\subsubsection{Sampling methods}

Several methods have been used in building energy analysis (including random sampling, Latin hypercube sampling, and Sobol sequence) to obtain the combinations of input variable values from probability density functions.

The random sampling method (also called traditional Monte Carlo) selects random samples from user-specified probability distributions [117-119]. Compared to other sampling methods, this method requires a large number of samples for convergence although it yields unbiased 
estimates of the mean and variance of outputs [120]. Asadi et al [121] implemented the Monte Carlo sampling method to generate 70,000 energy models with different input samples in order to analyse the effects of building shape on energy performance. Lu et al. [122] applied the random sampling method to estimate variations of electricity and gas consumption in Ma'anshan city of China using the Crystal ball software.

Latin hyper-cube sampling (LHS) is the most widely used sampling method in the field of building energy analysis as it can produce converged results with a considerably reduced number of samples $[3,51,52,58,59,107,111,116,123]$. LHS is a stratified sampling method that divides the range of every input variable into $\mathrm{N}$ segments (the specific sample size) with equal probability. The recommended length of the segments is dependent on the specified probability distribution shapes of input variables. One value is then randomly selected from each segment until all segments have one sample. An important feature in the LHS method is that every hypercube partitioned by segments associated with input variables has the same number of samples. Consequently, the LHS method usually requires a smaller sample size than the random sampling method for the same statistical accuracy. Hence, the LHS method is more suitable for computationally expensive models that include many uncertain variables. The general recommendation for a LHS sample size is ten times the number of variables in computer experiments [124]. However, this sample size may be inadequate for uncertainty analysis when complicated non-linear relationships between inputs and outputs exist in the building energy model. Further research is needed to provide clear guidance on the sampling number suitable for building energy analysis.

The quasi-Monte Carlo sampling method is an efficient space-filling technique to produce low discrepancy sequences by filling the unit hypercube with good uniformity of coverage. Several low-discrepancy sequences exist, including Halton, Sobol, and Faure [125]. Tian et al. [17] implemented the Sobol sequence for the identification of the key variables among ten input variables with respect to their effect on the energy use of an office building. Eisenhower et al. [126] applied the quasi-random sampling method for uncertainty and sensitivity analysis of building energy models. A comparison of Latin Hypercube and quasi Monte Carlo sampling methods was conducted by Kucherenko et al. [127].

In the case of correlated variables, several methods are available to maintain the correlation structure among variables, including the Iman/Conover, dependence-tree copula, and Stein method [36]. The Stein method has been used to consider the correlations between solar heat gain coefficients and U-values in generating input samples [128].

\subsubsection{Create and run energy models}

A large number of simulation runs are usually required in uncertainty analysis. Hence, it is necessary to automate the generation of simulation models with different input values, the simulation of models, and the extraction of the relevant data from simulation results using computer programming languages or specialized tools.

Several simulation programs have been used in uncertainty analysis of building energy use, including EnergyPlus [72, 111, 113, 117, 126, 127, 129, 130], ESP-r [6], TRNSYS [52, 118], SAP 2009 [131], DOE-2 [112, 121], and VA 114 [3]. EnergyPlus is the most widely used simulation software for the uncertainty analysis of building energy because the Input Data File (IDF) file required for EnergyPlus is an ASCII (i.e. text) file, which can be easily edited using computer languages [132]. The ISO 13790 monthly method is also used in forward uncertainty analysis of building energy [59] since it is able to rapidly compute many simulation runs using the simplified model. An additional module has been added in the ESP-r program to facilitate the application of Monte-Carlo based uncertainty analysis [44]. 
General-purpose programming languages can be used to edit building energy programs to automatically create many building energy models, including Matlab [3], Python [121], Excel VBA [2, 133], and R environment [134, 135]. Tian and de Wilde [2] used the Excel visual basic application (VBA) to create a large number of EnergyPlus models to investigate the impact of climate change on building thermal performance. Tian et al. [136] constructed approximately 100,000 EnergyPlus models using the R program based on the shape files of geographical information (GIS) data to study the energy performance of London Westminster area.

Researchers have developed special programing environments for the uncertainty analysis of building energy [37, 137]. The jEPlus program was developed by Zhang Yi [137] to support parametric analysis for EnergyPlus. This tool was used to draw samples from uncertainty ranges, create associated EnergyPlus models and simulate the models for uncertainty analysis of building energy models $[51,130]$. Recently, the GURA-W was developed to provide an extensive list of uncertainty sources, standard uncertainty quantification (UQ) repository, and advanced statistical methods for uncertainty and sensitivity analysis [37]. A key feature of the GURA-W is the UQ repository that houses the pre-defined distributions of uncertainty sources, which can be used as prior density functions for the common modelling practice if no additional detailed data are available on a building under investigation [138]. The GURA-W environment has been used to perform uncertainty analyses for sizing HVAC systems [31] and supporting performance-based contracts [56].

\subsubsection{Present uncertainty results}

Uncertainty in probabilistic model predictions can be presented as numerical values or as visual graphs. Numerical indicators are descriptive statistical measures including mean, median, standard deviation, percentile, interquartile range, coefficient of variation, and quantile. Typical graphical methods include histogram $[3,35,107,116,118]$, density plot $[13,59,107]$, cumulative distribution functions [56, 116, 122, 131], and box plots [16, 113]. Presently uncertainty analysis results is more complex if analysis results are multi-dimensional as a function of time or space. Time-series energy results are very common in assessing dynamic building energy behaviour. Box plots are a good method to show the variations of time series energy data. An example of this application is available in Tian et al. [2] to show the uncertainty of energy use in four time periods.

\subsubsection{Conduct sensitivity analysis}

Sensitivity analysis can be applied after presenting the results of output uncertainty analysis (Figure 2) in order to explain how input variables contribute to output variables [139]. While uncertainty and sensitivity analysis is closely related, they belong to two different disciplines. Uncertainty analysis is focused on either assessing output uncertainty derived from input uncertainty (i.e. forward uncertainty) or obtaining input uncertainty from measurement data (i.e. inverse uncertainty), whereas sensitivity analysis concentrates on assessing the contributions of input factors for variations of system outputs. Sensitivity analysis can be divided into local and global sensitivity analysis [36]. Detailed descriptions on the application of sensitivity analysis in building performance assessment can be found in $[36,134]$. DominguezMunoz et al. [116] used the SRC to determine the key factors affecting peak cooling loads. Tian and de Wilde [2] applied the SRC and Adaptive COmponent Selection and Smoothing Operator (ACOSSO) sensitivity methods to identify the important variables influencing energy performance of a campus building located in Plymouth, UK. Menberg et al. [140] developed an enhanced Morris method that uses the median value of analysis results instead of the average 
value for estimation of parameter importance, and demonstrated that the enhanced Morris method provides more robust results with a much reduced number of samples.

\subsection{Variations of Monte-Carlo methods}

This section describes three variations of Monte-Carlo uncertainty analysis that have been applied in building energy assessment. The first variation is two-dimensional Monte-Carlo method that allows aleatory and epistemic uncertainty to be treated differently in uncertainty propagation. The second variation is an incremental sampling method that generate several independent sets of Monte Carlo samples in order to obtain stable uncertainty results for building thermal performance. The third variation involves replacing time-consuming energy models with statistical surrogate models based on machine learning methods.

\subsubsection{Two dimensional Monte-Carlo methods}

Two-dimensional (2-D) Monte Carlo simulation is the most straightforward approach for propagating aleatory and epistemic uncertainty in a different manner. This method is also named the second-order probability, double loop, two-stage, 2-D Monte Carlo, or nested Monte Carlo method [70, 141, 142]. The outer loop simulates epistemic uncertainty, whereas the inner loop represents aleatory uncertainty. The advantage of using this method is that the uncertainty in model predictions arising from epistemic and aleatory uncertainty can be shown separately although its computational cost is usually very high due to the two-loop sampling. Figure 3 shows the probabilistic energy predictions of two example cases using this method. A single cumulative distribution function (CDF) denotes uncertainty in the predictions due to the aleatory uncertainty of input variables, while the spread of CDFs represents uncertainty in the predictions due to the epistemic uncertainty of input factors. In the case shown in Figure 3a, the aleatory uncertainty has a more dominate role in model outputs than the epistemic uncertainty, and the opposite trend is shown in the case in Figure $3 \mathrm{~b}$.

De Wilde and Tian [70] implemented the two-dimensional Monte Carlo approach to assess the impact of climate change on building thermal performance in a UK office building. In their study, the outer loop has 30 realizations using the random Monte Carlo sampling method and the inner loop generates 80 realizations per outer loop sample using the Latin Hypercube sampling method. Therefore, 2400 building energy models were run for one future climate scenario.

\subsubsection{Incremental sampling method}

The incremental sampling method is also called the replicate sampling method as it simply generates several independent samples in order to assess the stability of uncertainty results from the Monte Carlo sampling-based methods [110, 139]. First, an original sample is obtained using the random or LHS method. Then, the second sample is generated by the same method. The second sample is used to evaluate the adequacy of the original sample by comparing the probabilistic predictions from the original and second samples. If the results from the two samples are sufficiently similar, then the probabilistic predictions resulting from the samples represent the theoretical probability distribution. However, if the results from the two samples significantly differ, more samples are needed to ensure the convergence of probabilistic outcomes. The advantage of using the incremental LHS method is that it maintains the stratification of each LHS sample, which implies that several independent samples can be merged to provide more reliable uncertainty results. Note that the sizes of the samples can differ. This flexibility renders this method more useful by starting with a small sample size (to 
reduce computational cost) and then increasing the size of the next sample. Janssen [143] combined the incremental Latin hypercube sampling method with the sample-splitting approach to assess the accuracy of Monte Carlo simulations in the probabilistic design of a natural ventilating house.

\subsubsection{Surrogate model-based Monte Carlo method}

As discussed in section 4.1, forward uncertainty propagation requires many simulation runs to obtain reliable results. Hence, it may be infeasible or computationally challenging to use dynamic engineering-based models in uncertainty analysis. This has motivated the development of surrogate models that capture the main features of high-fidelity mathematical models while being efficient for both forward and inverse uncertainty quantification. Surrogate models can be categorized into two types: data-fit models and reduced-order models [144]. Data-fit models are based on regression methods to derive the relationships between inputs and outputs from a set of simulation results from high-fidelity models. Reduced-order models are generated by projecting high-dimensional states and parameters onto reduced-dimensional sub-spaces. Methods commonly used for the construction of reduced-order models are eigenfunctions, snapshotbased methods, and high-dimensional model representation (HMDR) methods, all of which are used to construct reduced base functions. Detailed mathematical descriptions of the methods can be found in $[8,144]$.

Data-fit models have been widely used in the building simulation domain, the most commonly used of which are linear regression models that have been considered as an effective surrogate model to capture building energy behaviour [1, 13, 121, 130]. In order to capture nonlinear behaviour, Chen et al. [111] constructed the MARS models to emulate daylighting simulation models and airflow network models for passively designed domestic buildings. Gelder et al. [145] provided an overview of five data-fit models (i.e., polynomial regression, MARS, kriging, radial basis function networks, and neural networks) and compared the performance of these methods through a case study. Recently, Gaussian Process models have been used as emulators for building simulation models due to their ability to flexibly capture complex behaviour including multivariable interactions and nonlinear relationships $[9,115$, 146].

\subsection{Non-sampling uncertainty propagation}

Non-sampling methods include perturbation methods, most probable point-based methods, operator-based methods, generalized polynomial chaos (stochastic Galerkin and collocation) and classical stochastic differential equations $[8,103,104]$. Perturbation methods can tackle large and complex parameterized models by expanding the random field through Taylor series at the most second-order expansion. The disadvantage of the perturbation approach is its limitation in handling large uncertainty in both inputs and outputs [103]. Most probable point-based methods are suitable for efficient uncertainty analysis using the first-order and second-order reliability methods [104, 147]. Operator-based methods are used to manipulate stochastic operators, for instance, by expressing the inverse of the stochastic operator in Neumann expansion [103]. Classical stochastic differential equations usually deal with idealized processes, including Wiener processes and Poisson processes using stochastic calculus [148, 149]. In contrast, generalized polynomial chaos methods allow inputs to be treated as random variables [103]. Generalized polynomial chaos, which has become a very popular method in uncertainty quantification, is a classical polynomial chaos in which the stochastic solutions are expressed as orthogonal polynomials of input variables. This method shows rapid convergence if the expanded function is dependent on smooth random variables. A Galerkin projection is usually 
implemented to minimize the error of the finite-order expansion and existing computer codes must be changed accordingly. For complex applications, an alternative choice is stochastic collocation that combines the advantage of both sampling-based (e.g. nonintrusive) and Galerkin methods (e.g. fast convergence) [148].

Non-sampling uncertainty propagation methods have been used only for a few of applications in the field of building energy analysis: stochastic different equations [150-153] and stochastic collocation [115, 154]. Brohus et al. [150] implemented stochastic differential equations to quantify the uncertainty of building energy consumption using two case studies. The first case is a mechanically ventilated building and the second case is a naturally ventilated atrium. The building loads are treated as stochastic processes with a time-varying mean value and a time-varying standard deviation using a white noise (e.g. stochastic part). The results indicate that the stochastic method can suitably describe the airflow and energy consumption although the computational time is high (several hours on an Athlon $1 \mathrm{GHz}$ PC in this study). Kim [115] created a surrogate model using polynomial chaos expansion for a five-storey office building located in South Korea. The point-collocation polynomial chaos method is used in MATLAB due to its non-intrusive nature. The results suggest that the polynomial chaos surrogate model is excellent in stochastic model predictions. Note that the stochastic collocation method can be used not only as a surrogate model to replace computationally expensive models but also as a sampling method for uncertainty quantification, which is the focus in this section. The uncertainty propagation using stochastic collocation has the advantage of fast convergence compared to the Monte Carlo sampling-based method [8]. However, further research, especially on the newly developed polynomial chaos expansion, is required to assess the suitability of the non-sampling uncertainty quantification in building energy analysis.

\subsection{Non-probabilistic uncertainty propagation}

A number of non-probabilistic uncertainty analysis methods are emerging to quantify uncertainty given limited information, especially for epistemic uncertainty. Non-probabilistic methods include interval analysis, fuzzy theory, Dempster-Shafer evidence theory, and the affine arithmetic model [27, 102, 155].

The simplest method in non-probabilistic uncertainty propagation is the interval analysis assuming that an interval scalar consists of a single continuous domain in the domain of real numbers bounded by a lower and an upper number [102]. Although the optimal solution (i.e. minimal and maximal values of output values due to input intervals) is difficult to find, this method is conceptually simple. Local and global optimization can be used to overcome this limitation, and an alternative method involves using a uniform distribution over the input intervals, which means that no values in this interval are equally likely. Note that the resulting outputs cannot be interpreted as a probabilistic distribution but should be treated as the output interval bounds. One of the problems with using the interval analysis is the dependency issue that leads to unreliable outputs for uncertainty analysis. The affine arithmetic model is a generalization of interval arithmetic by considering the correlation between variables [155]. The affine model is a linear transformation of uncertainty variables as a separate token [156]. Fuzzy set can be regarded as a direct extension from interval analysis. A fuzzy number is defined as a membership to describe the vagueness in the numeric value [44]. The most frequently used membership functions are the triangular and Gaussian probability densities [102]. The DempterShafer evidence theory is a generalization of classical probability theory to model input variables as the sets of intervals (e.g. one or more intervals). The computational cost of using this method can be very expensive and a surrogate model (as described in section 4.2.3) can be used to reduce the computational cost. 
Only a number of limited of studies have proposed non-probabilistic uncertainty methods in the area of building energy analysis [44, 156-158]. Macdonald and Clarke [156] implemented the affine arithmetic model in the energy conservation equations of the ESP-r simulation environment. The results indicate that this method not only reduces the computational efforts but also allows the flexibility of algorithm control in order to decrease output uncertainty. Chaney et al. [157] used the Dempster-Shafer evidence theory for the fusion of multiple-sensor data in a house simulation model. The advantage of Dempster-Shafer theory is that the data can be combined from multiple sources in which these data can be overlapping, contiguous, or even have gaps. They found that the evidence theory is a very reasonable approach for providing rich information about occupant interaction with systems in the house. Kim et al. [159] reported that the Demster-Shafer theory (DST) can be used to effectively combine five different experts' epistemic uncertainty into single uncertainty. The DST is known as a generalization of the subjective probability theory based on two principles. In the first principle, the degrees of belief from subjective probabilities are obtained, and the second principle is the aggregation rule (e.g. Dempster's rule, Yager's rule) for combining multiple degrees of belief. As energy efficiency projects often suffer from the limited information, more studies are needed to focus on finding ways to apply these non-probabilistic methods in order to more effectively represent the limited uncertainty information of building parameters.

\section{Inverse uncertainty quantification in building performance analysis}

Inverse uncertainty quantification is used to infer unknown input parameters in a model through a mathematical formulation given measurement data. Observed system outputs can be formulated as $[8,160]$,

$$
\mathrm{E}=\mathrm{f}(\mathrm{x}, \theta)+\delta+\varepsilon
$$

where $\mathrm{E}$ is the real observations (i.e. building energy data), $\mathrm{x}$ is the known parameters (such as monitored weather data), $\theta$ is the unknown parameters that need to be calibrated, $f(x, \theta)$ is the model outcomes predicted by the building energy model or the emulator, $\delta$ is the model discrepancy that capture the differences between predicted and actual building performances, and $\varepsilon$ is the measurement errors. Statistical methods for inverse uncertainty quantification can be broadly categorized into frequentist and Bayesian approaches. Prior to inverse uncertainty analysis, sensitivity analysis is often used to select important variables because it is difficult and computationally inefficient to infer a large number of uncertain parameters in model calibration.

\subsection{Frequentist techniques}

The frequentist technique is a classical parameter estimation approach that solely relies on measured data to infer unknown parameters [161-165]. This approach assumes that unknown parameters have true, fixed values and, accordingly, produce a single estimate and associated deviation. For linear models, unknown parameter estimates and associated confidence intervals can be determined explicitly based on normality assumption [8]. However, for nonlinearly parameterized problems, numerical optimization techniques are required, such as stochastic optimization methods, gradient-based approaches, and hybrid methods [8].

The most commonly used point estimation methods are maximum likelihood estimation (MLE) and least squares estimation (LSE). MLE computes a probability density function that compares measurements with model predictions using testing parameter values and yields parameter values that maximise the function. LSE is a special case of the MLE that calculates the weighted sum of the squares of the differences between measurements and model predictions. A key difference between the two methods is that LSE assumes that measurement errors are normally distributed whereas MLE handles a non-Gaussian error distribution. 
Andersen et al. [151] applied MLE for the estimation of building envelope-related parameters in the grey-box model of a building. Reddy and Andersen [167] compared different classical estimation methods, including MLE and LSE, through a case study in which parameter values in different chiller models are estimated based on hourly measurements from a chiller. The main issue of using these inverse methods is that the confidence intervals of parameters are not presented explicitly in detail. For linear models, confidence intervals of inferred parameters can be derived by most of the available statistical programs. Estimation of confidence intervals in non-linear models is more difficult, and please refer to [8] for detailed description.

Another way to quantify the uncertainty in parameter estimation is the bootstrapping technique [17, 168]. Based on the research from Banks et al. [168], the bootstrapping method is a better choice for a complex system because the sensitivities required for asymptotic theory are too complicated to compute for constant variance data. For non-constant variance data, local variation in the data would determine the choice of bootstrapping or asymptotic theory.

\subsection{Bayesian techniques}

Bayesian techniques have been increasingly used in estimating unknown parameters in building energy models [169-173]. The key feature of Bayesian methods is that expert knowledge can be incorporated with measurements into the model calibration process. In the Bayesian approach, unknown parameters are assigned with prior distributions that quantify prior beliefs about true parameter values based on expert knowledge collectively derived from a pool of available data sources such as surveys, technical reports, and industry standards. Prior distributions are updated using measurements through a Bayes' theorem in which the likelihood of matching observations with model prediction drives the updating process. This updating process combines prior knowledge with new observed information and leads to improved distributions of unknown parameters, also known as posterior distributions.

Ideally, when a large number of find-resolution observations are available, Bayesian calibration methods will result in posterior distributions that are close to true values regardless of initial prior beliefs about true parameter values. In reality, however, available observed data are often aggregated to the whole building level and are insufficient to infer a set of uncertain parameters. The effects of prior estimates on calibration outcomes have been investigated by increasing the range of prior estimates and altering the distribution shape to a uniform distribution in comparison to the original triangular distributions with tighter uncertainty ranges [46]. This comparative study showed that, while the change in the distribution shape has a more substantial effect on calibration results than the change in the distribution range, all cases nevertheless showed the same trend of updates from given prior estimates. An urban data analysis study based on Bayesian inference that disaggregated total energy use data into energy use per building type also found that while posterior distributions were significantly influenced by the choice of the prior distributions, they shifted toward the same position when they were considerably updated from the prior distributions [174].

Bayesian approaches have been formulated to calibrate a set of unknown parameters in the energy model with consideration of measurement errors [13, 35, 175] . In addition to measurement errors, the discrepancy between the model and the reality was recognised as another major uncertainty source. In order to account for the model discrepancy, Heo et al. [9] proposed a Bayesian approach that accounts for three types of uncertainties: (a) parameter uncertainty, (b) model discrepancy, and (c) observation errors. The approach (as shown in Figure 4) follows the mathematical formulation developed by Kennedy and O'Hagan [160] that uses the Gaussian process (GP) models to emulate a computer model and to capture differences between model predictions and observations due to the inability of the simulation model to represent the real behaviour. The Bayesian calibration method was demonstrated to substantially 
reduce uncertainty in posterior distributions that correspond well to true values under three levels of uncertainty consistent with different audit levels [4].

Using GP models as part of the calibration process is recognised as computationally expensive when the dimension number of calibration parameters or the number of data points is large. In order to alleviate the computational burden, Li et al. [176] proposed the application of a linear regression emulator that includes the main parameter and parameter interaction effects for predicting monthly energy consumptions. Through a case study, the linear model emulator was demonstrated to correctly capture the behaviour of the simulation model and yields similar calibration results to those when using GP models, with a significantly reduced computational time. Tian and Choudhary [13] also showed the suitability of using a linear regression model for predicting annual energy consumptions and employed a linear regression model as an emulator to calibrate a typical school building model against a collection of annual energy data of schools. In another study [35], a cooling capacity degradation model was used in the calibration process to estimate the ageing effect on the chiller maximum cooling capacity with the use of measured water temperature and flowrate data.

Since posterior distributions cannot be analytically derived for building energy models, Markov Chain Monte Carlo (MCMC) methods have been used to approximate theoretical posterior probability distributions by randomly drawing samples through the parameter space. Metropolis and Metropolis-Hastings algorithms are the most commonly used MCMC methods for computation of posterior distributions. These algorithms generate a random walk by sampling a proposed point from a jumping distribution based on the current point in an iterative manner and accepts the proposed point when it satisfies an acceptance criterion [177]. The choice of the jumping distribution substantially influences the number of points required to converge to the target distribution. Consequently, the algorithms often take a long time to move toward the high-probability density region by inefficiently exploring the entire parameter space. A Hamiltonian Monte Carlo method has been proposed and demonstrated to improve the inefficiency of the MCMC method by adding an auxiliary variable that enables faster movement through the parameter space [177]. It has been demonstrated by Menberg et al. [178] and Chong et al. [179] that this method substantially improved convergence speed in comparison to the random walk MCMC method for calibration of building energy models.

\section{Application of uncertainty analysis in building performance assessment}

This section discusses four applications in which the relevance of uncertainty analysis is demonstrated: building stock analysis, HVAC system sizing, variations of sensitivity index, and optimization under uncertainty. It should be noted that there are many types of applications where uncertainty analysis is useful in building energy analysis. The four applications are selected in this section to demonstrate the ways of implementing the uncertainty analysis techniques in building energy assessment. Table 3 shows the references for the four types of applications.

\subsection{Building stock analysis}

Significantly less research has been performed on building stock in comparison with individual buildings. One of the major reasons is high uncertainty/heterogeneity in input parameters for building stock analysis $[180,181]$. In order to overcome lack of the available data, uncertainty analysis has been implemented in recent research to incorporate possible variations of parameter values in assessing the energy performance of building stock.

Forward uncertainty analysis has been used in building stock usually to obtain the distributions of energy performance by varying unknown parameter values in the building stock 
model. Hughes et al. [131] used the forward Monte-Carlo uncertainty analysis method to generate the distribution of England's domestic energy consumption in 2010 by propagating input uncertainty, including individual dwelling type, dwelling age, and indoor set-point temperature in the total stock model. They emphasized the importance of using global sensitivity analysis methods to correctly identify key influential parameters. Kavgic et al. [118] applied the Monte Carlo method to predict probabilistic energy consumptions for space heating of the housing stock in Belgrade. The results demonstrate that the uncertainty in influential variables should be considered for the energy analysis of the building stock as it leads to large uncertainty in energy predictions. Li et al. [182] introduced coupling thermal and airflows methods to simulate the potential of natural ventilation of building stock in northern China.

Inverse analysis has been used to estimate the unknown parameters of energy models in a given building stock based on actual energy data [170, 183]. Tian and Choudhary [13] used the MCMC method in the OpenBUGS program to infer input distributions of the key variables influencing gas use in London secondary schools. They pointed out that the availability of building stock information is the key to selecting a suitable method for building stock energy models. The inverse method proposed in their study is suitable to make full use of the available energy data in order to improve the reliability of the building stock model. Tian et al. [19] used the R stan program, a new probabilistic language of full Bayesian statistical inference with Hamiltonian Monte Carlo, to estimate the locally varying energy use intensity in the domestic building stock of London. The results suggest this Bayesian hierarchical model can provide more reliable results compared to the geographically weighted regression method.

\subsection{HVAC system sizing}

Several studies have focused on sizing HVAC system using uncertainty quantification $[35,56,116,138]$. Peak heating/cooling loads of HVAC systems are usually calculated based on standardized design day data using the deterministic method [26], which does not provide sufficient and transparent information about the possible range of building loads and their likelihoods. Dominguez-Munoz et al [116] proposed a probabilistic approach to consider the uncertainty of input factors influencing peak cooling loads. Twenty uncertain factors are considered to calculate peak cooling loads using a simple lumped heat-balance model. Sun et al. [56] further explored the HVAC sizing issues for both heating and cooling loads using uncertainty analysis, which can replace the safety factor commonly used in HVAC industry. This uncertainty analysis include five groups of uncertainty sources: meteorological weather, microclimate, building, system, and occupant. Their analysis framework uses multiple actual year data instead of the design day method, which provides rich probabilistic information on heating/cooling demands to support risk-based sizing. The framework includes sensitivity analysis to find important factors influencing heating/cooling loads. Huang et al. [35] also proposed a probabilistic method for HVAC system design using multiple performance indicators (e.g., economic, energy, user satisfaction, and environmental criteria). Kim et el. al. [107] presented a multi-criterion stochastic decision making process for selecting the optimal HVAC system using the Bayesian Markov chain Monte Carlo method. In this study, Bayesian inference was used to obtain unknown quantities (expected utilities and weighting factors) in the formulation of a multi-attribute utility function. The Bayesian method provides a formal platform to quantify subjective preferences of diverse decision makers in a probabilistic manner and to mitigate the ambiguity of the multi-criterion decision making problem that involves multiple stakeholders. 


\subsection{Indicator of sensitivity analysis}

Uncertainty analysis can be used to estimate the variations of a sensitivity index affecting building thermal performance in order to provide more reliable and robust sensitivity analysis [184]. Assessing uncertainty of sensitivity analysis in the field of building energy analysis is still uncommon [17]. Bootstrap can be used to compute the variations of sensitivity index for ranking factors influencing building energy by a random sampling with replacement from an original dataset. Tian et al. [17] described the implementation of the bootstrap technique in detail for building energy analysis. Chen et al. [111] applied the bootstrap approach to obtain the confidence intervals of SRRC in assessing the thermal performance of a high-rise residential building.

\subsection{Optimization under uncertainty}

Optimization under uncertainty, which is also called robust design optimization (RDO) or reliability based design optimization (RBDO), is used to maximize the responses of a system (e.g. mean or median) while minimizing the system variability of responses (e.g. variance or standard deviation) [185]. In contrast, the deterministic optimization is only focused on maximizing the system responses. Building energy performance is affected by a number of uncertain factors, such as weather variations, occupant behaviour, and randomness of thermal properties. Hence, the optimal design solution derived by the deterministic approach may not be the optimal choice in reality when all uncertainty sources impact building performance. Optimization under uncertainty is not a new challenge in engineering problems. However, only very limited studies are available on optimization under uncertainty in building energy analysis [21, 22, 186-188].

Gang et al. [189] developed an uncertainty-based optimization method for the design of district cooling systems. In their study, variables are classified into three groups: outdoor weather, building design/construction, and indoor conditions. Through sensitivity analyses, they show that indoor conditions are the most important factor influencing the performance of district cooling systems, while the variations in building design/construction have the least influence. Hopfe et al. [190] performed the S metric selection-evolutionary multi-objective optimisation algorithm (SMS-EMOA) for robust optimisation of building design. To reduce the computational burden, a Kriging meta-model was created to replace computationally expensive energy models. A case study building located in the Netherlands was used to demonstrate the suitability of this method of combining the meta-model and SMS-EMOA. Kim et al. [188] presented a multi-criterion stochastic optimal selection of a double glazing system for an office building. In their study, LHS samplings and stochastic objective function were used with the GP emulator, genetic algorithm and Pareto optimality for stochastic performance optimization.

\section{Software for uncertainty analysis}

The section firstly describes the software used in forward and inverse uncertainty analysis of building energy use as shown in Table 4. Then the software for construction of surrogate models and sensitivity analysis in building environment is discussed since these two methods are closely linked to uncertainty analysis as described in sections 4.1 .5 and section 4.2.3, respectively.

Forward uncertainty analysis needs computer programming in three steps: obtain the sampling of input parameters, create and run building energy models, and present and summarise probabilistic results. The programming languages for creating and running building energy models were described in section 4.1.3. Simlab, a free development framework for 
sensitivity and uncertainty analysis [191], has been widely used in building energy analysis as it provides a number of sampling methods, including the random sample, Latin hyper-cube sampling, and Sobol method [3, 52, 59, 123, 128]. R environment provides many sampling methods, such as Latin hyper-cube [192], Sobol sequence [193], and Halton sequence [193]. Crystal ball, a spreadsheet-based environment for risk analysis [194], has been used in uncertainty analysis of building energy use [122]. Another excellent computing environment for uncertainty analysis is the Dakota developed by USA Sandia National Laboratories that has advanced abilities to handle mixed epistemic-aleatory uncertainty, optimization under uncertainty, and Bayesian calibration [195]. The R mc2d package can be easily used to run 2-D Monte Carlo simulations [196]. Analyst also offers their own computing procedure for 2-D Monte Carlo simulations based on 1-D Monte Carlo methods.

Inverse uncertainty analysis requires more complicated computation, especially for Bayesian computation. The Bayesian inference Using Gibbs Sampling (BUGS) programs (WinBugs or OpenBugs) provide a flexible software environment for the Bayesian analysis using the MCMC method [197] to infer unknown parameters [13, 198]. The R computing environment also provides the interface for the BUGS programs [199] and the Gaussianprocess-based Bayesian method [200] (as described in section 5.2). The R stan package [201] supports full Bayesian statistical inference with the advanced computation method, Hamiltonian Monte Carlo, which was used to compute the distribution of energy use intensities in London in a more efficient way [19].

Surrogate models have been used to expedite the computation of both forward and inverse uncertainty analysis in building energy assessment. $\mathrm{R}$ environment has many good packages for machine learning computation and an overview of these methods is available in $\mathrm{R}$ task view [202]. A meta package of Classification and Regression Training (caret) [203] is particularly useful because it combines a large number of machine learning methods to easily create several machine learning models and compare their predictive performance. Matlab also provides a number of machine learning methods and a helpful reference book has also been presented [204].

Sensitivity analysis is often used together with uncertainty analysis as discussed in section 4.1. R sensitivity package [205] offers several global sensitivity analysis methods, including standardised regression coefficients, Morris screen method, Sobol indices, extended Fourier amplitude sensitivity test, and kriging-based sensitivity analysis. The Simlab program [191] mainly contains a set of global sensitivity analysis based on sampling-based methods, such as standardized (rank) regression coefficient, Morris method, and Sobol sensitivity indicators. The Simlab program has been used widely in building energy analysis [72, 113, 114, 134]. The latest version of Simlab 4 provides a closer link to the $R$ statistical environment [191].

\section{Conclusions and further work}

In this paper, the research progress of uncertainty analysis in building energy assessment was reviewed from the following four aspects. The state-of-art research development of data sources for uncertain parameters relevant to building energy performance were firstly described. The next two sections discussed the detailed methods of both forward and inverse uncertainty quantification in building energy analysis. The third aspect of this review involved the four types of applications in implementing uncertainty analysis when assessing building performance. For the final aspect, the software available that can be used in uncertainty analysis of building energy use were described. The research trends of uncertainty analysis when assessing thermal performance in buildings from this overview are summarized as follows.

(1) Previous research on data sources for uncertainty analysis relevant to building energy performance has provided a firm foundation for developing different types 
of uncertainty analysis. The construction of a database of uncertainty quantification, such as the GURA-W workbench, would be beneficial to provide transparent and robust uncertainty quantification for the whole community of building energy simulation.

(2) Occupant behaviour is a complicated factor that has a significant impact on building energy performance. Implicit models are likely to remain dominant in simulating the variations of occupant behaviour in the near future.

(3) Among the forward uncertainty quantification methods, the sampling-based Monte Carlo method is the most widely used forward uncertainty method in the field of building energy assessment because this method is intuitive and only requires running energy models a number of times.

(4) Recent research has focused on Bayesian inverse computation to infer unknown parameters in building energy models because this method can incorporate the prior information on unknown parameters from previous studies, site surveys, and industry standards.

(5) Specialized tools (such as Matlab, R, and jEPlus) have been developed to generate samples from specified uncertainty distributions and to create and run a large number of building energy models for uncertainty analysis.

Uncertainty analysis has been ready to become a mainstream method in assessing building thermal performance from this overview because the relevant statistical methods are mature and the sufficient applications of these methods in building energy analysis are becoming available. More connection between the fundamentals of uncertainty quantification and the features of building energy analysis should be built to provide more flexible analysis for achieving sustainable high-performance buildings. The recommendations for further research on uncertainty analysis of building energy are presented as follows.

- More effort still needs to be placed on rigorously quantifying the uncertainty of input parameters, which is regarded as the most difficult task in ensuring the quality of uncertainty analysis results. The databases for quantifying uncertainty input parameters need to be constructed in terms of various indicators, such as building types, climate characteristics, and new or existing buildings.

- More attention should be paid to better simulate stochastic occupant behaviour and interactions with other systems in buildings by using both implicit and explicit occupant models.

- For the sampling-based methods, further studies are required to provide clear guidance on the sampling size to provide converged probabilistic outcomes for building energy analysis.

- Further research on the 2-D Monte Carlo method is required since this approach can be used to represent both aleatory and epistemic uncertainty in building energy assessment. New visualization methods should be explored to show the complicated uncertainty results.

- Both non-sampling and non-probabilistic uncertainty methods are useful as alternative forward uncertainty quantification approaches when only very limited information is available. Further research is needed to suitably apply these methods in building energy assessment.

- Many issues still need to be addressed in applying Bayesian inverse computation. It is important to clearly understand how the availability of energy use data in combination with prior beliefs specified as prior distributions affects the posterior distributions of input variables inferred from Bayesian computation. Many new methods (such as Hamiltonian Monte Carlo) have not been sufficiently explored to test the relevance in calibrating building energy models. 
- Considering a number of uncertain factors at the design stage, further research on optimization under uncertainty (robust design) is required for low-energy buildings.

\section{ACKNOWLEDGEMENT}

This research was supported by the National Natural Science Foundation of China (No. 51778416) and the Key Projects of Philosophy and Social Sciences Research, Ministry of Education (China) "Research on Green Design in Sustainable Development" (contract No. 16JZDH014, approval No. 16JZD014). The research was also supported by the Program for Innovative Research Team in University of Tianjin (No. TD13-5012/5045).

\section{References:}

[1] Tian W, Yang S, Li Z, Wei S, Pan W, Liu Y. Identifying informative energy data in Bayesian calibration of building energy models. Energy and Buildings. 2016;119:363-76. [2] Tian W, de Wilde P. Uncertainty and sensitivity analysis of building performance using probabilistic climate projections: A UK case study. Automation in Construction. 2011;20:1096109.

[3] Hopfe CJ, Hensen JLM. Uncertainty analysis in building performance simulation for design support. Energy and Buildings. 2011;43:2798-805.

[4] Heo Y, Graziano DJ, Guzowski L, Muehleisen RT. Evaluation of calibration efficacy under different levels of uncertainty. Journal of Building Performance Simulation. 2015;8:135-44.

[5] de Wit S, Augenbroe G. Analysis of uncertainty in building design evaluations and its implications. Energy and Buildings. 2002;34:951-8.

[6] Macdonald I, Strachan P. Practical application of uncertainty analysis. Energy and Buildings. 2001;33:219-27.

[7] Arendt PD, Apley DW, Chen W. Quantification of model uncertainty: Calibration, model discrepancy, and identifiability. Journal of Mechanical Design. 2012;134:100908.

[8] Smith RC. Smith R C. Uncertainty quantification: theory, implementation, and applications. London: SIAM; 2013.

[9] Heo Y, Choudhary R, Augenbroe GA. Calibration of building energy models for retrofit analysis under uncertainty. Energy and Buildings. 2012;47:550-60.

[10] Almeida RM, Ramos NM, Manuel S. Towards a methodology to include building energy simulation uncertainty in the Life Cycle Cost analysis of rehabilitation alternatives. Journal of Building Engineering. 2015;2:44-51.

[11] Cabeza LF, Rincón L, Vilariño V, Pérez G, Castell A. Life cycle assessment (LCA) and life cycle energy analysis (LCEA) of buildings and the building sector: A review. Renewable and Sustainable Energy Reviews. 2014;29:394-416.

[12] Hoxha E, Habert G, Lasvaux S, Chevalier J, Le Roy R. Influence of construction material uncertainties on residential building LCA reliability. Journal of Cleaner Production.

2017;144:33-47.

[13] Tian W, Choudhary R. A probabilistic energy model for non-domestic building sectors applied to analysis of school buildings in greater London. Energy and Buildings. 2012;54:1-11. [14] Tian W, Choudhary R, Augenbroe G, Lee SH. Importance analysis and meta-model construction with correlated variables in evaluation of thermal performance of campus buildings. Building and Environment. 2015;92:61-74.

[15] de Wilde P, Tian W. Towards probabilistic performance metrics for climate change impact studies. Energy and Buildings. 2011;43:3013-8.

[16] Tian W, de Wilde P. Thermal building simulation using the UKCP09 probabilistic climate projections. Journal of Building Performance Simulation. 2011;4:105-24. 
[17] Tian W, Song J, Li Z, de Wilde P. Bootstrap techniques for sensitivity analysis and model selection in building thermal performance analysis. Applied Energy. 2014;135:320-8.

[18] Tian W, Liu Y, Heo Y, Yan D, Li Z, An J, et al. Relative importance of factors influencing building energy in urban environment. Energy. 2016;111:237-50.

[19] Tian W, Yang S, Meng Q, Wei L. Spatial Variation of Urban Building Energy Analysis.

IBPSA Building Simulation Conference, December 7-9, 2015. Hyderabad, India. 2015.

[20] Choudhary R, Tian W. Influence of district features on energy consumption in nondomestic buildings. Building Research \& Information. 2014;42:32-46.

[21] Rezvan AT, Gharneh NS, Gharehpetian GB. Robust optimization of distributed generation investment in buildings. Energy. 2012;48:455-63.

[22] Ramallo-González AP, Blight TS, Coley DA. New optimisation methodology to uncover robust low energy designs that accounts for occupant behaviour or other unknowns. Journal of Building Engineering. 2015;2:59-68.

[23] Beven K. Environmental modelling: An uncertain future?: CRC Press; 2010.

[24] Friswell MI, Fonseca JR, Mottershead JE, Lees AW. Quantification of uncertainty using inverse methods. Proceedings of the 45th AIAA/ASME/ASCE/AHS/ASC Structures, Structural Dynamics and Materials Conference, 19-22 April 2004, . Palm Springs, California, USA2004. p. 1756-63.

[25] Hayes K. Uncertainty and uncertainty analysis methods. Australian Centre of Excellence for Risk Assessment (ACERA) project A. 2011;705.

[26] ASHRAE. Handbook of Fundamentals, Atlanta: American Society of Heating, AirConditioning and Refrigeration Engineers. Inc; 2013.

[27] Swiler LP, Paez TL, Mayes RL. Epistemic uncertainty quantification tutorial. Proceedings of the 27th International Modal Analysis Conference, February 9-12, 2009 Orlando, Florida USA. 2009.

[28] Helton JC. Conceptual and computational basis for the quantification of margins and uncertainty. Sandia National Laboratories (United States). Funding organisation: US Department of Energy (United States); 2009.

[29] de Wit S. Uncertainty in building simulation. In: Malkawi A, Godfried Augenbroe, editors. Advanced building simulation: Routledge; 2004. p. 25-59.

[30] Van Gelder L, Janssen H, Roels S. Probabilistic design and analysis of building performances: Methodology and application example. Energy and Buildings. 2014;79:202-11. [31] Wang Q. Accuracy, validity and relevance of probabilistic building energy models: Georgia Institute of Technology; 2016.

[32] Sun Y. Closing the building energy performance gap by improving our predictions:

Georgia Institute of Technology; 2014.

[33] Silva AS, Ghisi E. Uncertainty analysis of user behaviour and physical parameters in residential building performance simulation. Energy and Buildings. 2014;76:381-91.

[34] Struck C, J. H. On supporting design decisions in conceptual design addressing specification uncertainties using performance simulation. The 10th IBPSA Building Simulation Conference (BS2007), September 03-06, 2007, . Beijing, China. 2007.

[35] Huang P, Huang G, Wang Y. HVAC system design under peak load prediction uncertainty using multiple-criterion decision making technique. Energy and Buildings. 2015;91:26-36.

[36] Tian W. A review of sensitivity analysis methods in building energy analysis. Renewable and Sustainable Energy Reviews. 2013;20:411-9.

[37] Lee B, Sun Y, Augenbroe G, Paredis C. Towards better prediction of building performance: a workbench to analyze uncertainty in building simulation. Proceedings of building simulation 2013. Sydney2013.

[38] de Wilde P. The gap between predicted and measured energy performance of buildings: A framework for investigation. Automation in Construction. 2014;41:40-9. 
[39] Clarke JA, Hensen JLM. Integrated building performance simulation: Progress, prospects and requirements. Building and Environment. 2015;91:294-306.

[40] Webster J, Watson RT. Analyzing the Past to Prepare for the Future: Writing a Literature Review. MIS Quarterly. 2002;26:xiii-xxiii.

[41] Wei Y, Zhang X, Shi Y, Xia L, Pan S, Wu J, et al. A review of data-driven approaches for prediction and classification of building energy consumption. Renewable and Sustainable Energy Reviews. 2018;82:1027-47.

[42] Amasyali K, El-Gohary NM. A review of data-driven building energy consumption prediction studies. Renewable and Sustainable Energy Reviews. 2018;81:1192-205.

[43] Koulamas C, Kalogeras AP, Pacheco-Torres R, Casillas J, Ferrarini L. Suitability analysis of modeling and assessment approaches in energy efficiency in buildings. Energy and Buildings. 2018; 158:1662-82.

[44] Macdonald IA. Quantifying the effects of uncertainty in building simulation [Doctor]: University of Strathclyde; 2002.

[45] Wit Sd. Uncertainty in predictions of thermal comfort in buildings: \{Sun, 2014 \#944\}; 2001.

[46] Heo Y. Bayesian calibration of building energy models for energy retrofit decision-making under uncertainty: Georgia Institute of Technology; 2011.

[47] Struck C. Uncertainty propagation and sensitivity analysis techniques in building performance simulation to support conceptual building and system design: Eindhoven University of Technology; 2012.

[48] Eames M, Ramallo-Gonzalez A, Wood M. An update of the UK's test reference year: The implications of a revised climate on building design. Building Services Engineering Research and Technology. 2016;37:316-33.

[49] Hong T, Chang W-K, Lin H-W. A fresh look at weather impact on peak electricity demand and energy use of buildings using 30-year actual weather data. Applied Energy. 2013;111:33350 .

[50] Kershaw T, Eames M, Coley D. Comparison of multi-year and reference year building simulations. Building Services Engineering Research and Technology. 2010;31:357-69.

[51] Calleja Rodríguez G, Carrillo Andrés A, Domínguez Muñoz F, Cejudo López JM, Zhang Y. Uncertainties and sensitivity analysis in building energy simulation using macroparameters. Energy and Buildings. 2013;67:79-87.

[52] Breesch H, Janssens A. Performance evaluation of passive cooling in office buildings based on uncertainty and sensitivity analysis. Solar Energy. 2010;84:1453-67.

[53] Yoo H, Lee K, Levermore GJ. Comparison of heating and cooling energy simulation using multi-years and typical weather data in South Korea. Building Services Engineering Research and Technology. 2015;36:18-33.

[54] Hui SC, Cheung K. Multi-year (MY) building simulation: is it useful and practical. Proc of the IBPSA Building Simulation'97 Conference (BS1997), September 8-10, 1997,. Prague,

Czech Republic, 1997.

[55] Wang L, Mathew P, Pang X. Uncertainties in energy consumption introduced by building operations and weather for a medium-size office building. Energy and Buildings. 2012;53:1528.

[56] Sun Y, Gu L, Wu CFJ, Augenbroe G. Exploring HVAC system sizing under uncertainty. Energy and Buildings. 2014;81:243-52.

[57] Lee BD, Sun Y, Hu H, Augenbroe G, Paredis CJ. A framework for generating stochastic meteorological years for risk-conscious design of buildings. Proceedings of the SimBuild 2012 Conference, August 1-3, 2012, . Madison, Wisconsin, USA2012. p. 345-52. 
[58] Sun Y, Heo Y, Tan M, Xie H, Jeff Wu C, Augenbroe G. Uncertainty quantification of microclimate variables in building energy models. Journal of Building Performance Simulation. 2014;7:17-32.

[59] Corrado V, Mechri HE. Uncertainty and sensitivity analysis for building energy rating. Journal of building physics. 2009;33:125-56.

[60] Braun MR, Altan H, Beck SBM. Using regression analysis to predict the future energy consumption of a supermarket in the UK. Applied Energy. 2014;130:305-13.

[61] Du H, Underwood C, Edge J. Generating design reference years from the UKCP09 projections and their application to future air-conditioning loads. Building Services Engineering Research and Technology. 2012;33:63-79.

[62] Eames M, Kershaw T, Coley D. On the creation of future probabilistic design weather years from UKCP09. Building Services Engineering Research and Technology. 2010.

[63] Belcher S, Hacker J, Powell D. Constructing design weather data for future climates.

Building Services Engineering Research and Technology. 2005;26:49-61.

[64] Zhu M, Pan Y, Huang Z, Xu P. An alternative method to predict future weather data for building energy demand simulation under global climate change. Energy and Buildings.

2016;113:74-86.

[65] Murphy J, Sexton D, Jenkins G, Boorman P, Booth B, Brown K, et al. UKCP09 Climate change projections. UK Climate Impacts Programme,[Online] Accessed 18th August. 2010.

[66] Clarke J A YPP, Pinney A A. The harmonization of thermal properties of building materials. BRE, UK; 1990.

[67] Domínguez-Muñoz F, Anderson B, Cejudo-López JM, Carrillo-Andrés A. Uncertainty in the thermal conductivity of insulation materials. Energy and Buildings. 2010;42:2159-68.

[68] R. OD, L. HS. Faber \& Kell 's Heating and Air-Conditioning of Buildings: Elsevier; 2008.

[69] Thevenard D, Haddad K. Ground reflectivity in the context of building energy simulation.

Energy and Buildings. 2006;38:972-80.

[70] de Wilde P, Tian W. Identification of key factors for uncertainty in the prediction of the thermal performance of an office building under climate change. Building simulation.

2009;2:157-74.

[71] Wang Q, Augenbroe G, Sun Y. The role of construction detailing and workmanship in achieving energy efficient building. Construction Research Congress. Atlanta, USA2014. p. 1924.

[72] Tian W, Wilde PD. Impact of global warming on thermal performance of domestic buildings using probabilistic climate data. International Journal of Global Warming. 2016;10:514-35.

[73] Emmerich S J, McDowell T P, W A. Investigation of the impact of commercial building envelope airtightness on HVAC energy use, US Department of Commerce, Technology Administration, National Institute of Standards and Technology, Report NISTIR 7238. 2005.

[74] K PA. Myths about building envelopes. ASHRAE Journal. 1999;41:39-48.

[75] Li H, Li X, Qi M. Field testing of natural ventilation in college student dormitories (Beijing, China). Building and Environment. 2014;78:36-43.

[76] HJ M. Assessing mold risks in buildings under uncertainty: Georgia Institute of Technology; 2005.

[77] Smith A, Luck R, Mago PJ. Analysis of a combined cooling, heating, and power system model under different operating strategies with input and model data uncertainty. Energy and Buildings. 2010;42:2231-40.

[78] Yan B, Li X, Malkawi AM, Augenbroe G. Quantifying uncertainty in outdoor air flow control and its impacts on building performance simulation and fault detection. Energy and Buildings. 2017;134:115-28. 
[79] Griffith B, Long N, Torcellini P, Judkoff R, Crawley D, Ryan J. Methodology for modeling building energy performance across the commercial sector, Technical Report NREL/TP-55041956, March 2008, National Renewable Energy Laboratory, USA. Golden, CO, National Renewable Energy Laboratory2008.

[80] de Wilde P, Tian W, Augenbroe G. Longitudinal prediction of the operational energy use of buildings. Building and Environment. 2011;46:1670-80.

[81] Huang P, Wang Y, Huang G, Augenbroe G. Investigation of the ageing effect on chiller plant maximum cooling capacity using Bayesian Markov Chain Monte Carlo method. Journal of Building Performance Simulation. 2016;9:529-41.

[82] Hong T, Taylor-Lange SC, D’Oca S, Yan D, Corgnati SP. Advances in research and applications of energy-related occupant behavior in buildings. Energy and Buildings. 2016;116:694-702.

[83] Yan D, O’Brien W, Hong T, Feng X, Burak Gunay H, Tahmasebi F, et al. Occupant behavior modeling for building performance simulation: Current state and future challenges. Energy and Buildings. 2015;107:264-78.

[84] Hoes P, Hensen JLM, Loomans MGLC, de Vries B, Bourgeois D. User behavior in whole building simulation. Energy and Buildings. 2009;41:295-302.

[85] Wei S, Jones R, de Wilde P. Driving factors for occupant-controlled space heating in residential buildings. Energy and Buildings. 2014;70:36-44.

[86] O'Brien W, Gunay HB. Mitigating office performance uncertainty of occupant use of window blinds and lighting using robust design. Building Simulation. 2015:1-16.

[87] Cecconi FR, Manfren M, Tagliabue LC, Ciribini ALC, De Angelis E. Probabilistic behavioral modeling in building performance simulation: A Monte Carlo approach. Energy and Buildings. 2017;148:128-41.

[88] Eguaras-Martínez M, Vidaurre-Arbizu M, Martín-Gómez C. Simulation and evaluation of Building Information Modeling in a real pilot site. Applied Energy. 2014;114:475-84.

[89] Fabi V, Andersen RV, Corgnati SP, Olesen BW. A methodology for modelling energyrelated human behaviour: Application to window opening behaviour in residential buildings. Building Simulation. 2013;6:415-27.

[90] Bahaj AS, James PAB. Urban energy generation: The added value of photovoltaics in social housing. Renewable and Sustainable Energy Reviews. 2007;11:2121-36.

[91] Gunay HB, O'Brien W, Beausoleil-Morrison I. A critical review of observation studies, modeling, and simulation of adaptive occupant behaviors in offices. Building and Environment. 2013;70:31-47.

[92] O'Neill Z, Niu F. Uncertainty and sensitivity analysis of spatio-temporal occupant behaviors on residential building energy usage utilizing Karhunen-Loève expansion. Building and Environment. 2017;115:157-72.

[93] Hong T, D'Oca S, Turner WJN, Taylor-Lange SC. An ontology to represent energy-related occupant behavior in buildings. Part I: Introduction to the DNAs framework. Building and Environment. 2015;92:764-77.

[94] Ward R, Choudhary R, Heo Y, Rysanek A. Exploring the impact of different parameterisations of occupant-related internal loads in building energy simulation. Energy and Buildings. 2016;123:92-105.

[95] Menezes AC, Cripps A, Buswell RA, Wright J, Bouchlaghem D. Estimating the energy consumption and power demand of small power equipment in office buildings. Energy and Buildings. 2014;75:199-209.

[96] Rysanek AM, Choudhary R. DELORES - an open-source tool for stochastic prediction of occupant services demand. Journal of Building Performance Simulation. 2015;8:97-118.

[97] Yao J. Determining the energy performance of manually controlled solar shades: A stochastic model based co-simulation analysis. Applied Energy. 2014;127:64-80. 
[98] Lee YS, Malkawi AM. Simulating multiple occupant behaviors in buildings: An agentbased modeling approach. Energy and Buildings. 2014;69:407-16.

[99] Haldi F, Robinson D. The impact of occupants' behaviour on building energy demand. Journal of Building Performance Simulation. 2011;4:323-38.

[100] Ahn K-U, Park CS. Different Occupant Modeling Approaches for Building Energy

Prediction. Energy Procedia. 2016;88:721-4.

[101] Ahn K-U, Park C-S. Correlation between occupants and energy consumption. Energy and Buildings. 2016;116:420-33.

[102] Moens D, Vandepitte D. A survey of non-probabilistic uncertainty treatment in finite element analysis. Computer Methods in Applied Mechanics and Engineering. 2005;194:152755.

[103] Xiu D. Fast Numerical Methods for Stochastic Computations: A Review.

Communications in computational physics. 2009;5:247-72.

[104] Lee SH, Chen W. A comparative study of uncertainty propagation methods for black-boxtype problems. Structural and Multidisciplinary Optimization. 2009;37:239-53.

[105] Bijl H, Lucor, D., Mishra, S., \& Schwab, C. Uncertainty quantification in computational fluid dynamics. London: Springer Science \& Business Media; 2013.

[106] Najm HN. Uncertainty Quantification and Polynomial Chaos Techniques in

Computational Fluid Dynamics. Annual Review of Fluid Mechanics. 2009;41:35-52.

[107] Kim Y-J, Ahn K-U, Park C-S. Decision making of HVAC system using Bayesian Markov chain Monte Carlo method. Energy and Buildings. 2014;72:112-21.

[108] Yi H, Braham WW. Uncertainty characterization of building emergy analysis (BEmA).

Building and Environment. 2015;92:538-58.

[109] Silva AS, Ghisi E. Uncertainty analysis of the computer model in building performance simulation. Energy and Buildings. 2014;76:258-69.

[110] Brian M. Adams MSE, Michael S. Eldred, John D. Jakeman,, Kathryn A. Maupin JAM, Laura P. Swiler, J. Adam Stephens,, Dena M. Vigil TMW. Dakota, A Multilevel Parallel Object-Oriented Framework for Design Optimization, Parameter Estimation, Uncertainty Quantification, and Sensitivity Analysis: Version 6.4 User's Manual. SAND2014-4633 Unlimited Release; 2016.

[111] Chen X, Yang H, Sun K. Developing a meta-model for sensitivity analyses and prediction of building performance for passively designed high-rise residential buildings. Applied Energy. 2016:422-39.

[112] Mottahedi M, Mohammadpour A, Amiri SS, Riley D, Asadi S. Multi-linear Regression Models to Predict the Annual Energy Consumption of an Office Building with Different Shapes. Procedia Engineering. 2015;118:622-9.

[113] Singh R, Lazarus IJ, Kishore VVN. Uncertainty and sensitivity analyses of energy and visual performances of office building with external venetian blind shading in hot-dry climate. Applied Energy. 2016;184:155-70.

[114] Tian W, Yang S, Zuo J, Li Z, Liu Y. Relationship between built form and energy performance of office buildings in a severe cold Chinese region. Building simulation. 2017;10:11-24.

[115] Kim Y-J. Comparative study of surrogate models for uncertainty quantification of building energy model: Gaussian Process Emulator vs. Polynomial Chaos Expansion. Energy and Buildings. 2016;133:46-58.

[116] Domínguez-Muñoz F, Cejudo-López JM, Carrillo-Andrés A. Uncertainty in peak cooling load calculations. Energy and Buildings. 2010;42:1010-8.

[117] Spitz C, Mora L, Wurtz E, Jay A. Practical application of uncertainty analysis and sensitivity analysis on an experimental house. Energy and Buildings. 2012;55:459-70. 
[118] Kavgic M, Summerfield A, Mumovic D, Stevanovic Z. Application of a Monte Carlo model to predict space heating energy use of Belgrade's housing stock. Journal of Building Performance Simulation. 2015;8:375-90.

[119] Prada A, Cappelletti F, Baggio P, Gasparella A. On the effect of material uncertainties in envelope heat transfer simulations. Energy and Buildings. 2014;71:53-60.

[120] Saltelli A, Tarantola S, Campolongo F, Ratto M. Sensitivity analysis in practice: a guide to assessing scientific models: Wiley; 2004.

[121] Asadi S, Amiri SS, Mottahedi M. On the development of multi-linear regression analysis to assess energy consumption in the early stages of building design. Energy and Buildings. 2014;85:246-55.

[122] Lu Y, Huang Z, Zhang T. Method and case study of quantitative uncertainty analysis in building energy consumption inventories. Energy and Buildings. 2013;57:193-8.

[123] Struck C, Hensen J, Kotek P. On the application of uncertainty and sensitivity analysis with abstract building performance simulation tools. Journal of building physics. 2009;33:5-27. [124] Levy S, Steinberg DM. Computer experiments: A review. AStA Advances in Statistical Analysis. 2010;94:311-24.

[125] Diwekar U, David A. Uncertainty Analysis and Sampling Techniques. BONUS Algorithm for Large Scale Stochastic Nonlinear Programming Problems. New York, NY: Springer New York; 2015. p. 9-25.

[126] Eisenhower B, O'Neill Z, Fonoberov VA, Mezić I. Uncertainty and sensitivity decomposition of building energy models. Journal of Building Performance Simulation. 2012;5:171-84.

[127] Kucherenko S, Albrecht D, Saltelli A. Comparison of Latin Hypercube and Quasi Monte Carlo Sampling Techniques. IBPSA (International Building Performance Simulation Association) conference 2011, November 14-16 Sydney2011.

[128] Lam TC, Ge H, Fazio P. Energy positive curtain wall configurations for a cold climate using the Analysis of Variance (ANOVA) approach. Building Simulation. 2016;9:297-310. [129] Evins R, Orehounig K, Dorer V. Variability between domestic buildings: the impact on energy use. Journal of Building Performance Simulation. 2016;9:162-75.

[130] Korolija I, Zhang Y, Marjanovic-Halburd L, Hanby VI. Regression models for predicting UK office building energy consumption from heating and cooling demands. Energy and Buildings. 2013;59:214-27.

[131] Hughes M, Palmer J, Cheng V, Shipworth D. Global sensitivity analysis of England's housing energy model. Journal of Building Performance Simulation. 2015;8:283-94.

[132] DOE. EnergyPlus V8.6, September 2016, Department of Energy, USA. 2016.

[133] de Wilde P, Tian W. Predicting the performance of an office under climate change: A study of metrics, sensitivity and zonal resolution. Energy and Buildings. 2010;42:1674-84. [134] Yang S, Tian W, Cubi E, Meng Q, Liu Y, Wei L. Comparison of Sensitivity Analysis Methods in Building Energy Assessment. Procedia Engineering. 2016;146:174-81.

[135] Wei L, Tian W, Zuo J, Yang Z, Yang S, Song J. Effects of Building Form on Energy Use for Buildings in Cold Climate Regions. Procedia Engineering. 2016;146:182-9.

[136] Tian W, Rysanek A, Choudhary R, Heo Y. High Resolution Energy Simulations At City Scale IBPSA Building Simulation Conference (BS2015), December 7-9, 2015. Hyderabad, India.2015.

[137] Y Z. Parallel EnergyPlus and the development of a parametric analysis The 11th Conference of International Building Performance Association IBPSA (BS2009), July 27-30, 2009, . Glasgow, UK, 2009.

[138] Chen J, Augenbroe G, Song X. Evaluating the potential of hybrid ventilation for small to medium sized office buildings with different intelligent controls and uncertainties in US climates. Energy and Buildings. 2018;158:1648-61. 
[139] Helton JC, Johnson JD, Sallaberry CJ, Storlie CB. Survey of sampling-based methods for uncertainty and sensitivity analysis. Reliability Engineering \& System Safety. 2006;91:1175209.

[140] Menberg K, Heo Y, Choudhary R. Sensitivity analysis methods for building energy models: Comparing computational costs and extractable information. Energy and Buildings. 2016;133:433-45.

[141] Nannapaneni S, Mahadevan S. Reliability analysis under epistemic uncertainty. Reliability Engineering \& System Safety. 2016;155:9-20.

[142] Tian W, Fu X, Sun Y, Yin B, Meng X, Liu Y. Sustainable Building Design Based on the Second Order Probability Approach. Procedia Engineering. 2017;205:1056-63.

[143] Janssen H. Monte-Carlo based uncertainty analysis: Sampling efficiency and sampling convergence. Reliability Engineering \& System Safety. 2013;109:123-32.

[144] Biegler L, Biros, G., Ghattas, O., Heinkenschloss, M., Keyes, D., Mallick, B. Large-scale inverse problems and quantification of uncertainty: John Wiley \& Sons; 2011.

[145] Van Gelder L, Das P, Janssen H, Roels S. Comparative study of metamodelling techniques in building energy simulation: Guidelines for practitioners. Simulation Modelling Practice and Theory. 2014;49:245-57.

[146] Manfren M, Aste N, Moshksar R. Calibration and uncertainty analysis for computer models - A meta-model based approach for integrated building energy simulation. Applied Energy. 2013;103:627-41.

[147] Du X, Chen W. A Most Probable Point-Based Method for Efficient Uncertainty Analysis. Journal of Design and Manufacturing Automation. 2001;4:47-66.

[148] Xiu D. Efficient collocational approach for parametric uncertainty analysis.

Communications in computational physics. 2007;2:293-309.

[149] Oksendal B. Stochastic differential equations: an introduction with applications: Springer Science \& Business Media; 2013.

[150] Brohus H, Frier C, Heiselberg P, Haghighat F. Quantification of uncertainty in predicting building energy consumption: A stochastic approach. Energy and Buildings. 2012;55:127-40.

[151] Andersen KK, Madsen H, Hansen LH. Modelling the heat dynamics of a building using stochastic differential equations. Energy and Buildings. 2000;31:13-24.

[152] Jiménez MJ, Madsen H, Bloem JJ, Dammann B. Estimation of non-linear continuous time models for the heat exchange dynamics of building integrated photovoltaic modules. Energy and Buildings. 2008;40:157-67.

[153] Jacob D, Burhenne S, Herkel S. Comparing two methods of stochastic modeling for buildings Proceedings of Building Simulation 2011: 12th Conference of International Building Performance Simulation Association, November 14-16, 2011, . Sydney, Australia, 2011.

[154] Fontanini A, Vaidya U, Ganapathysubramanian B. A stochastic approach to modeling the dynamics of natural ventilation systems. Energy and Buildings. 2013;63:87-97.

[155] Degrauwe D, Lombaert G, De Roeck G. Improving interval analysis in finite element calculations by means of affine arithmetic. Computers \& Structures. 2010;88:247-54.

[156] Macdonald IA, Clarke JA. Applying uncertainty considerations to energy conservation equations. Energy and Buildings. 2007;39:1019-26.

[157] Chaney J, Hugh Owens E, Peacock AD. An evidence based approach to determining residential occupancy and its role in demand response management. Energy and Buildings. 2016;125:254-66.

[158] Tian W, Meng X, Yin B, Sun Y, Liu Y, Fu X. Design of Robust Green Buildings Using a Non-probabilistic Uncertainty Analysis Method. Procedia Engineering. 2017;205:1049-55.

[159] Kim Y, Ahn K, Park C. Aggregated epistemic uncertainty for building energy prediction using Dempster-Shafer evidence theory. Proceedings of 9th IAQVEC (Indoor Air Quality 
Ventilation \& Energy Conservation In Buildings) Conference October 23-26, 2016. Incheon, Korea. 2016.

[160] Kennedy MC, O'Hagan A. Bayesian calibration of computer models. Journal of the Royal Statistical Society Series B, Statistical Methodology. 2001:425-64.

[161] Fumo N, Rafe Biswas MA. Regression analysis for prediction of residential energy consumption. Renewable and Sustainable Energy Reviews. 2015;47:332-43.

[162] Masuda H, Claridge DE. Statistical modeling of the building energy balance variable for screening of metered energy use in large commercial buildings. Energy and Buildings.

2014;77:292-303.

[163] Paulus MT, Claridge DE, Culp C. Algorithm for automating the selection of a temperature dependent change point model. Energy and Buildings. 2015;87:95-104.

[164] Aranda A, Ferreira G, Mainar-Toledo MD, Scarpellini S, Llera Sastresa E. Multiple regression models to predict the annual energy consumption in the Spanish banking sector. Energy and Buildings. 2012;49:380-7.

[165] Vu DH, Muttaqi KM, Agalgaonkar AP. A variance inflation factor and backward elimination based robust regression model for forecasting monthly electricity demand using climatic variables. Applied Energy. 2015;140:385-94.

[166] Omlin M, Reichert P. A comparison of techniques for the estimation of model prediction uncertainty. Ecological Modelling. 1999;115:45-59.

[167] Reddy TA, Andersen KK. An evaluation of classical steady-state off-line linear parameter estimation methods applied to chiller performance data. HVAC\&R Research. 2002;8:101-24.

[168] Banks HT, Holm K, Robbins D. Standard error computations for uncertainty quantification in inverse problems: Asymptotic theory vs. bootstrapping. Mathematical and Computer Modelling. 2010;52:1610-25.

[169] Booth AT, Choudhary R, Spiegelhalter DJ. Handling uncertainty in housing stock models. Building and environment. 2012;48:35-47.

[170] Heo Y, Augenbroe G, Graziano D, Muehleisen RT, Guzowski L. Scalable methodology for large scale building energy improvement: Relevance of calibration in model-based retrofit analysis. Building and Environment. 2015;87:342-50.

[171] Kang Y, Krarti M. Bayesian-Emulator based parameter identification for calibrating energy models for existing buildings. Building Simulation. 2016;9:411-28.

[172] Chong A, Lam KP, Pozzi M, Yang J. Bayesian calibration of building energy models with large datasets. Energy and Buildings. 2017;154:343-55.

[173] Yu D. A two-step approach to forecasting city-wide building energy demand. Energy and Buildings. 2018;160:1-9.

[174] Booth A, Choudhary R, Spiegelhalter D. A hierarchical Bayesian framework for calibrating micro-level models with macro-level data. Journal of Building Performance Simulation. 2013;6:293-318.

[175] Kim Y-J, Yoon S-H, Park C-S. Stochastic comparison between simplified energy calculation and dynamic simulation. Energy and Buildings. 2013;64:332-42.

[176] Li Q, Augenbroe G, Brown J. Assessment of linear emulators in lightweight Bayesian calibration of dynamic building energy models for parameter estimation and performance prediction. Energy and Buildings. 2016;124:194-202.

[177] Gelman A, Carlin JB, Stern HS, Rubin DB. Bayesian data analysis: Chapman \& Hall/CRC Boca Raton, FL, USA; 2014.

[178] Menberg K, Heo Y, Choudhary R. Efficiency and Reliability of Bayesian Calibration of Energy Supply System Models. IBPSA Building Simulation Conference (BS2017), August 7-9, 2017 San Francisco, USA. 2017. 
[179] Chong A, Lam KP. A Comparison of MCMC Algorithms for the Bayesian Calibration of Building Energy Models. IBPSA Building Simulation Conference (BS2017), August 7-9, 2017 San Francisco, USA. 2017.

[180] Fonseca JNB, Oliveira Panão MJN. Monte Carlo housing stock model to predict the energy performance indicators. Energy and Buildings. 2017;152:503-15.

[181] Naber E, Volk R, Schultmann F. From the Building Level Energy Performance Assessment to the National Level: How are Uncertainties Handled in Building Stock Models. Procedia Engineering. 2017;180:1443-52.

[182] Li Y, Li X. Natural ventilation potential of high-rise residential buildings in northern China using coupling thermal and airflow simulations. Building Simulation. 2015;8:51-64. [183] Zhao F, Lee SH, Augenbroe G. Reconstructing building stock to replicate energy consumption data. Energy and Buildings. 2016;117:301-12.

[184] Song J, Wei L, Sun Y, Tian W. Implementation of Meta-modelling for Sensitivity Analysis in Building Energy Analysis. The eSim 2014 conference, . May 7 to 10, Ottawa, Canada2014.

[185] Seshadri P, Constantine P, Iaccarino G, Parks G. A density-matching approach for optimization under uncertainty. Computer Methods in Applied Mechanics and Engineering. 2016;305:562-78.

[186] Nguyen A-T, Reiter S, Rigo P. A review on simulation-based optimization methods applied to building performance analysis. Applied Energy. 2014;113:1043-58.

[187] Das P, Van Gelder L, Janssen H, Roels S. Designing uncertain optimization schemes for the economic assessment of stock energy-efficiency measures. Journal of Building Performance Simulation. 2015:1-14.

[188] Kim Y-J, Park C-S. Multi-criterion stochastic optimal selection of a double glazing system. Building Simulation. 2017;10:1-9.

[189] Gang W, Augenbroe G, Wang S, Fan C, Xiao F. An uncertainty-based design optimization method for district cooling systems. Energy. 2016;102:516-27.

[190] Hopfe CJ, Emmerich MT, Marijt R, Hensen J. Robust multi-criteria design optimisation in building design. Proceedings of Building Simulation and Optimization. Loughborough, UK2012.

[191] Tarantola S, Becker W. SIMLAB Software for Uncertainty and Sensitivity Analysis. In: Ghanem R, Higdon D, Owhadi H, editors. Handbook of Uncertainty Quantification. Cham: Springer International Publishing; 2016. p. 1-21.

[192] Carnell R. R package lhs: Latin Hypercube Samples. R package version 0.14, https://CRAN.R-project.org/package=lhs, accessed 2016-11-10. 2016.

[193] Christophe D, Petr S. R package randtoolbox: Generating and Testing Random Numbers. $\mathrm{R}$ package version 1.17. https://CRAN.R-project.org/package=randtoolbox accessed 2016-0601.2015.

[194] Rose D. Risk analysis: a quantitative guide: John Wiley \& Sons; 2008.

[195] Swiler LP, Eldred MS, Adams BM. Dakota: Bridging Advanced Scalable Uncertainty Quantification Algorithms with Production Deployment. In: Ghanem R, Higdon D, Owhadi H, editors. Handbook of Uncertainty Quantification. Cham: Springer International Publishing; 2016. p. 1-43.

[196] Pouillot R, Delignette-Muller ML. Evaluating variability and uncertainty separately in microbial quantitative risk assessment using two R packages. International Journal of Food Microbiology. 2010;142:330-40.

[197] Thomas A, O'Hara B, Ligges U, Sturtz S. Making BUGS open. R news. 2006;6:12-7. [198] Choudhary R. Energy analysis of the non-domestic building stock of Greater London. Building and environment. 2012;51:243-54. 
[199] Marley JK, Wand MP. Non-standard semiparametric regression via BRugs. Journal of Statistical Software. 2010;37:1-30.

[200] Hankin RKS. Introducing BACCO, an R Bundle for Bayesian Analysis of Computer Code Output. 2005. 2005;14:21.

[201] Hoffman MD, Gelman A. The No-U-turn sampler: adaptively setting path lengths in Hamiltonian Monte Carlo. Journal of Machine Learning Research. 2014;15:1593-623.

[202] Hothorn T. CRAN Task View: Machine Learning \& Statistical Learning, https://CRAN.R-project.org/view=MachineLearning, accessed on 2016-11-10. 2016.

[203] Kuhn M, Johnson K. Applied predictive modeling: Springer; 2013.

[204] Murphy KP. Machine learning: a probabilistic perspective: MIT press; 2012.

[205] Gilles Pujol BI, Alexandre Janon. R package sensitivity V1.12.2: sensitivity: Global Sensitivity Analysis of Model Outputs. https://CRAN.R-project.org/package=sensitivity accessed on 2016-11-20. 2016. 